\title{
CONSTRAINED OPTIMAL CONTROL THEORY FOR DIFFERENTIAL LINEAR REPETITIVE PROCESSES*
}

\author{
M. DYMKOV ${ }^{\dagger}$, E. ROGERS ${ }^{\ddagger}$, S. DYMKOU ${ }^{\S}$, AND K. GALKOWSKI
}

\begin{abstract}
Differential repetitive processes are a distinct class of continuous-discrete twodimensional linear systems of both systems theoretic and applications interest. These processes complete a series of sweeps termed passes through a set of dynamics defined over a finite duration known as the pass length, and once the end is reached the process is reset to its starting position before the next pass begins. Moreover the output or pass profile produced on each pass explicitly contributes to the dynamics of the next one. Applications areas include iterative learning control and iterative solution algorithms, for classes of dynamic nonlinear optimal control problems based on the maximum principle, and the modeling of numerous industrial processes such as metal rolling, long-wall cutting, etc. In this paper we develop substantial new results on optimal control of these processes in the presence of constraints where the cost function and constraints are motivated by practical application of iterative learning control to robotic manipulators and other electromechanical systems. The analysis is based on generalizing the well-known maximum and $\epsilon$-maximum principles to them.
\end{abstract}

Key words. two-dimensional systems, optimal control, constraints

AMS subject classifications. 93C05, 93C15

DOI. $10.1137 / 060668298$

1. Introduction. Repetitive processes are a distinct class of two-dimensional (2D) systems of both systems theoretic and applications interest. The unique characteristic of such a process is a series of sweeps, termed passes, through a set of dynamics defined over a fixed finite duration known as the pass length. On each pass an output, termed the pass profile, is produced which acts as a forcing function on, and hence contributes to, the dynamics of the next pass profile. This, in turn, leads to the unique control problem in that the output sequence of pass profiles generated can contain oscillations which increase in amplitude in the pass-to-pass direction.

To introduce a formal definition, let $\alpha<+\infty$ denote the pass length (assumed constant). Then in a repetitive process the pass profile $y_{k}(t), 0 \leq t \leq \alpha$, generated on pass $k$ acts as a forcing function on, and hence contributes to, the dynamics of the next pass profile $y_{k+1}(t), 0 \leq t \leq \alpha, k \geq 0$.

Physical examples of repetitive processes include long-wall coal cutting and metalrolling operations (see, for example, the references cited in [17]). Also in recent years applications have arisen where adopting a repetitive process setting for analysis has distinct advantages over alternatives. Examples of these so-called algorithmic applications include classes of iterative learning control (ILC) schemes (see, for example, [13]) and iterative algorithms for solving nonlinear dynamic optimal control problems based on the maximum principle [15]. In the case of iterative learning control for the linear

${ }^{*}$ Received by the editors August 25, 2006; accepted for publication (in revised form) September 18, 2007; published electronically January 30, 2008.

http://www.siam.org/journals/sicon/47-1/66829.html

${ }^{\dagger}$ Belarus State Economic University, Partizanski Ave., 26, Minsk, Belarus (dymkov_m@bseu.by).

${ }^{\ddagger}$ Corresponding author. School of Electronics and Computer Science, University of Southampton, Southampton SO 17 1BJ, UK (etar@ecs.soton.ac.uk).

$\S$ Department of Applied Mathematics II, Friedrich-Alexander-University of Erlangen-Nuremberg, Martensstrasse 3, 91058 Erlangen, Germany (dymkou@am2.am.uni-erlangen.de).

IInstitute of Control and Computation Engineering, University of Zielona Góra, Podgorna 50, 65-246, Zielona Góra, Poland (K.Galkowski@issi.uz.zgora.pl).

396 
dynamics case, the stability theory for differential (and discrete) linear repetitive processes is one method which can be used to undertake a stability/convergence analysis of a powerful class of such algorithms and thereby produce vital design information concerning the trade-offs required between convergence and transient performance (see, for example, [14]).

Attempts to control these processes using standard (or 1D) systems theory and associated algorithms fail (except in a few very restrictive special cases) precisely because such an approach ignores their inherent 2D systems structure, i.e., information propagation occurs from pass to pass and along a given pass. Also the initial conditions are reset before the start of each new pass, and the structure of these can be somewhat complex. For example, if they are an explicit function of points on the previous pass profile, then this alone can destroy stability. In seeking a rigorous foundation on which to develop a control theory for these processes, it is natural to attempt to exploit structural links which exist between these processes and other classes of $2 \mathrm{D}$ linear systems.

The case of $2 \mathrm{D}$ discrete linear systems recursive in the positive quadrant $(i, j)$ : $i \geq 0, j \geq 0$ (where $i$ and $j$ denote the directions of information propagation) has been the subject of much research effort over the years using, in the main, the well-known Roesser [16] and Fornasini-Marchesini [8] state-space models. One approach which has been the subject of productive research is optimal control - see, for example, $[4$, 18]. More recently, productive research has been reported on $\mathcal{H}_{\infty}$ and $\mathcal{H}_{2}$ approaches to analysis and controller design - see, for example, $[19,7]$. In this paper we consider so-called differential linear repetitive processes where information propagation along the pass is governed by a matrix differential equation. The systems theory for $2 \mathrm{D}$ discrete linear systems is therefore not applicable. (Also, as noted above, for discrete processes the resetting and structure of the boundary conditions may cause problems which have no Roesser or Fornasini-Marchesini state-space model counterparts.)

In this paper we develop substantial new results on the optimal control of differential linear repetitive processes with constraints which we motivate from the iterative learning control application. The results themselves are obtained by extending the maximum principle and the $\epsilon$-maximum principle [11] to them. A sensitivity analysis of the resulting optimal control is also undertaken, and some relevant differentiation properties are established. Finally, a numerical example is given.

2. Preliminaries. ILC is a technique for controlling systems which are required to continually repeat the same operation with the requirement that a reference trajectory defined over a finite interval is followed to a high precision. In particular, the system completes a pass (also known as a trial in some literature) and is then reset, the next pass is completed, and so on. The basic idea of ILC is to use information from previous executions of the task in order to improve performance from pass to pass in the sense that the tracking error is sequentially reduced. It is clear therefore that ILC can easily be formulated as a repetitive process, and the stability theory for them can be used to explain why an incorrectly designed ILC scheme can result in nonconvergent behavior which manifests itself as oscillations that increase in amplitude from pass to pass [14].

Since the original work in the mid-1980s [3], the general area of ILC has been the subject of considerable research in terms of the underlying theory (with experimental verification in some cases). Commonly used ILC algorithms construct the input to the plant or process from the input used on the last pass plus an additive increment, which is typically a function of the past values of the measured output error, i.e., 
the difference between the achieved output on the current pass and the desired plant output. Suppose that $u_{k}(t)$ denotes the input to the plant on pass $k$ which is of duration $\alpha$, i.e., $0 \leq t \leq \alpha<\infty$. Suppose also that $e_{k}(t)=r(t)-y_{k}(t)$ denotes the current pass error. Then the objective of constructing a sequence of input functions such that the performance achieved is gradually improving with each successive pass can be refined to a convergence condition on the input and error, i.e.,

$$
\lim _{k \rightarrow \infty}\left\|e_{k}\right\|=0, \quad \lim _{k \rightarrow \infty}\left\|u_{k}-u_{\infty}\right\|=0
$$

where $\|\cdot\|$ is a signal norm in a suitably chosen function space with a norm-based topology and $u_{\infty}$ is termed the learned control.

A large number of design algorithms have been developed for this general area, some of which have also been experimentally tested. Of these, a good number are based on minimization of a cost function. Given the tracking nature of this general problem in the pass-to-pass direction, it is clearly necessary to penalize control action to prevent a "large" error resulting in the demand for an unacceptably high control input on the next pass in an attempt to minimize the error. One class of such algorithms is termed norm-optimal (with an extension to so-called predictive norm-optimal which is not relevant here). Here (see [1] for full details) on completion of pass $k$, the control input for pass $k+1$ is computed as the solution of the minimum norm optimization problem

$$
u_{k+1}=\arg \min _{u_{k+1}}\left\{J_{k+1}\left(u_{k+1}\right): e_{k+1}=r-y_{k+1}, y_{k+1}=G u_{k+1}\right\},
$$

where the performance index, or optimality criterion, used is defined to be

$$
J_{k+1}\left(u_{k+1}\right)=\left\|e_{k+1}\right\|_{\mathcal{Y}}^{2}+\left\|u_{k+1}-u_{k}\right\|_{\mathcal{U}}^{2},
$$

where $\mathcal{Y}$ is a real Hilbert space of output or pass profile signals $\left(y_{k}\right)$ and $\mathcal{U}$ is a real (and possibly distinct) Hilbert space of input signals $\left(u_{k}\right)$. Here the initial control $u_{0} \in \mathcal{U}$ can be arbitrary in theory but, in practice, will be a good first guess at the solution of the problem. This problem can be interpreted as the determination of the control input on pass $k+1$ with the properties that: (i) the tracking error is reduced in an optimal way; and (ii) this new control input does not deviate too much from the control input used on pass $k$. The relative weighting of these two objectives can be absorbed into the definitions of the norms in $\mathcal{Y}$ and $\mathcal{U}$. Other approaches to learning control in the presence of input constraints can be found in, for example, [9, 20] (but note that repetitive processes appear in formulations which have no iterative learning control interpretation). Suppose now that the plant dynamics are described by the following matrix differential equation:

$$
\frac{d x_{k}(t)}{d t}=A x_{k}(t)+D x_{k-1}(t)+b u_{k}(t), \quad 0 \leq t \leq \alpha, \quad k \geq 0,
$$

where, on pass $k, x_{k}(t)$ is the $n \times 1$ state (equal to the pass profile or output) vector, $u_{k}(t)$ is the scalar control input, $A, D$ are constant $n \times n$ matrices, and $b$ is a given $n \times 1$ vector. (This model is chosen for simplicity of presentation and is easily extended to the case when the pass profile vector is a linear combination of the current pass state, input, and previous pass profile vectors.)

Then it is straightforward to show that the above formulation includes the choice of a linear quadratic cost function as a special case, but the solution has to be modified 
slightly to guarantee that the resulting Riccati equation-based solution is causal in the sense that it does not, as the dynamics evolve, require use of information which is not yet available - see [1] for the details here. Algorithms resulting from this approach have been experimentally tested on a chain conveyor system with, on the whole, very encouraging results [2]. However, in some cases it was observed that the computed control input (a scalar variable in this application) was still above the safe operating range of the actuator device and the experiment had to be stopped to prevent damage. Also there was a tendency for the output at the end of each pass to "dip down" in value.

Another feature of repetitive processes which does not appear in the above optimal control problem is that in each practical application only a finite number of passes will actually be completed. Suppose therefore that $N<\infty$ denotes the number of passes actually completed, introduce the set $K=\{1,2, \ldots, N\}$, and let $T$ denote the finite interval (the pass length) $[0, \alpha]$. Then, with the above observations in mind, consider (1) with boundary conditions

$$
x_{k}(0)=d_{k}, \quad k \in K, \quad x_{0}(t)=f(t), \quad t \in T,
$$

where $d_{k}$ is an $n \times 1$ vector with constant entries and $f(t)$ is a known function $t \in T$. Then the optimal control problem considered is

$$
\max _{u_{k}} J(u), J(u)=\sum_{k \in K} p_{k}^{T} x_{k}(\alpha),
$$

where $p_{k}, k=1, \ldots, N$, is a given $n \times 1$ vector subject to an end of pass (or terminal) constraint of the form

$$
H_{k} x_{k}(\alpha)=o_{k}, \quad k \in K,
$$

where $o_{k}$ is an $m \times 1$ vector and $H_{k}$ is an $m \times n$ matrix, and the control inputs satisfy the following admissibility condition.

DEFINITION 1. For each pass number $k \in K$ the piecewise continuous function $u_{k}: T \rightarrow R$ is termed an admissible control for this pass if it satisfies

$$
\left|u_{k}(t)\right| \leq 1, t \in T
$$

and the corresponding state vector $x_{k}(t), t \in T$, of (1) satisfies the boundary conditions

$$
x_{k}(0)=d_{k}, H_{k} x_{k}(\alpha)=o_{k} .
$$

Also, without loss of generality, we assume that the matrix $A$ has simple eigenvalues $\lambda_{i}, 1 \leq i \leq n$, and that it is stable in the sense that $\operatorname{Re} \lambda_{i}<0,1 \leq i \leq n$. (Stability of the matrix $A$ is a necessary condition for so-called stability along the pass (essentially bounded input bounded output stability) independent of the pass length [17].)

3. Optimality conditions for the supporting control functions. Consider first (1)-(2) in the absence of the terminal conditions (4). Then it has been shown elsewhere [5] that the solution of these equations can be written as

$$
\begin{aligned}
x_{k}(t)= & \sum_{j=1}^{k} K_{j}(t) d_{k+1-j}+\int_{0}^{t} K_{k}(t-\tau) D f(\tau) d \tau \\
& +\sum_{j=1}^{k} \int_{0}^{t} K_{j}(t-\tau) b u_{k+1-j}(\tau) d \tau, k=1, \ldots, N,
\end{aligned}
$$

Copyright (c) by SIAM. Unauthorized reproduction of this article is prohibited. 
where the $K_{i}(t)$ are the solutions of the following $n \times n$ matrix differential equations:

$$
\dot{K}_{1}(t)=A K_{1}(t), \quad \dot{K}_{i}(t)=A K_{i}(t)+D K_{i-1}(t), \quad i=2, \ldots, N,
$$

with initial conditions

$$
K_{1}(0)=I_{n}, \quad K_{i}(0)=0, \quad i=2, \ldots, N .
$$

Also it is easy to show that these solutions have the following properties:

$$
\begin{array}{ll}
K_{j}(t-\sigma)=\int_{\sigma}^{t} K_{j-k}(t-\tau) D K_{k}(\tau-\sigma) d \tau, & 0 \leq \sigma<t \leq \alpha, \quad k=1, \ldots, j-1, \\
K_{j}(t-\sigma)=\sum_{s=1}^{j} K_{s}(t-\tau) K_{j+1-s}(\tau-\sigma), & j=2, \ldots, N-1,
\end{array}
$$

which will be used below.

Now by using (6) we can rewrite the optimal problem considered here in the following integral form:

$$
\max _{u_{1}, \ldots, u_{N}} J(u), J(u)=\sum_{j=1}^{N} \int_{0}^{\alpha} c_{j}(\tau) u_{j}(\tau) d \tau+\gamma,
$$

subject to the terminal conditions (4) and the control constraint (5). Also we can write

$$
\begin{array}{r}
\int_{0}^{\alpha} g_{11}(\tau) u_{1}(\tau) d \tau=h_{1}, \\
\int_{0}^{\alpha}\left[g_{21}(\tau) u_{1}(\tau)+g_{22}(\tau) u_{2}(\tau)\right] d \tau=h_{2}
\end{array}
$$

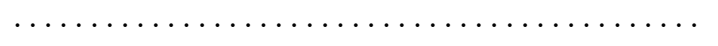

and

$$
\int_{0}^{\alpha}\left[g_{N 1}(\tau) u_{1}(\tau)+\cdots+g_{N N}(\tau) u_{N}(\tau)\right] d \tau=h_{N}
$$

$$
\left|u_{k}(\tau)\right| \leq 1, \tau \in T, \quad k=1, \ldots, N,
$$

where the scalar $\gamma$ and the scalar functions $c_{j}(\tau)$ are defined as follows:

$$
\begin{aligned}
\gamma & =\sum_{k=1}^{N} \sum_{j=1}^{k} p_{k}^{T} K_{j}(\alpha) d_{k+1-j}+\sum_{k=1}^{N} \int_{0}^{\alpha} p_{k}^{T} K_{k}(\alpha-\tau) D f(\tau) d \tau, \\
c_{j}(\tau) & =\sum_{k=j}^{N} p_{k}^{T} K_{k+1-j}(\alpha-\tau) b, \quad j=1, \ldots, N, \quad g_{k j}(\tau)=H_{k} K_{k+1-j}(\alpha-\tau) b, j \leq k, \\
h_{k} & =o_{k}-\sum_{j=1}^{k} H_{k} K_{j}(\alpha) d_{k+1-j}-\int_{0}^{\alpha} H_{k} K_{k}(\alpha-\tau) D f(\tau) d \tau, k=1, \ldots, N .
\end{aligned}
$$

Also we require the following.

Copyright (c) by SIAM. Unauthorized reproduction of this article is prohibited. 
Definition 2. For each fixed $k, 1 \leq k \leq N$, the time instances $\tau_{k i}, 1 \leq$ $i \leq m: 0<\tau_{k 1}<\tau_{k 2}<\cdots<\tau_{k m}<\alpha$ are termed supporting, and their collection $\tau_{\text {sup }}^{k}:=\left\{\tau_{k 1}, \ldots, \tau_{k m}\right\}$ is termed the support of pass $k$ for $(1)-(4)$ if the matrix $G_{\text {sup }}^{k}:=$ $\left\{g_{k k}\left(\tau_{k 1}\right), \ldots, g_{k k}\left(\tau_{k m}\right)\right\}$ (i.e., the $j$ th column of the matrix here is the $m \times 1$ vector $\left.g_{k k}\left(\tau_{k j}\right)\right)$ is nonsingular.

By using (7) we have that $g_{k k}(\tau)=H_{k} e^{A(\alpha-\tau)} b$. Therefore the existence of the support $\tau_{\text {sup }}^{k}$ is guaranteed by controllability of the pair $\left\{H_{k} A, b\right\}$.

Definition 3. A pair $\left\{\tau_{\text {sup }}^{k}, u_{k}(t), k=1, \ldots, N\right\}$ consisting of a support $\tau_{\text {sup }}^{k}$ and admissible control functions $u_{k}(t), t \in T$ is termed a supporting control function for (1)-(4).

Remark 1. These last two definitions are motivated as follows. Often an optimal control problem solution has the so-called bang-bang form; i.e., the control function takes only boundary values in the admissible set $U$. If $U=\{-1 \leq u \leq+1\}$, then $u^{0}(t)= \pm 1$ (the "switch-on/switch-off" regime). Also the switching times are constructive elements in the design of the optimal controller. Hence, our goal is to apply these key elements directly to the optimality conditions, and consequently we use the supporting time instances and control.

Let $\left\{\tau_{\text {sup }}^{k}, u_{k}(t), k=1, \ldots, N\right\}$ be a support control function and construct a sequence of $m \times 1$ vectors $\left\{\nu^{(k)}, k=1, \ldots, N\right\}$ by solving the following set of linear algebraic equations:

$$
\begin{aligned}
\left(\nu^{(N)}\right)^{T} G_{\text {sup }}^{N}-c_{\text {sup }}^{(N)} & =0, \\
\left(\nu^{(N-1)}\right)^{T} G_{\text {sup }}^{N-1}+\left(\nu^{(N)}\right)^{T} F_{(N-1) \text { sup }}^{N}-c_{\text {sup }}^{(N-1)} & =0, \\
\ldots \ldots \ldots \ldots \ldots \ldots \ldots \ldots \ldots \ldots \ldots \ldots \ldots \ldots \ldots \ldots \ldots \ldots \ldots \ldots \ldots \ldots & \\
\left(\nu^{(1)}\right)^{T} G_{\text {sup }}^{1}+\left(\nu^{(2)}\right)^{T} F_{1 \text { sup }}^{2}+\cdots+\left(\nu^{(N)}\right)^{T} F_{1 \text { sup }}^{N}-c_{\text {sup }}^{(1)} & =0,
\end{aligned}
$$

where the $1 \times m$ vectors $c_{\text {sup }}^{(k)}$ and the $m \times m$ matrices $F_{j s u p}^{k}$ are given by

$$
c_{\text {sup }}^{(k)}:=\left(c_{k}\left(\tau_{k 1}\right), \ldots, c_{k}\left(\tau_{k m}\right)\right), \quad k=1, \ldots, N,
$$

and

$$
F_{j s u p}^{k}:=\left(g_{k j}\left(\tau_{j 1}\right), \ldots, g_{k j}\left(\tau_{j m}\right)\right), \quad k>j, \quad j=1, \ldots, N-1,
$$

respectively.

Introduce the $1 \times m N$ vectors $(\hat{\nu})^{T}$ and $c_{\text {sup }}$ as

$$
(\hat{\nu})^{T}=\left(\left(\nu^{(1)}\right)^{T}, \ldots,\left(\nu^{(N)}\right)^{T}\right), \quad c_{\text {sup }}=\left(c_{\text {sup }}^{(1)}, c_{\text {sup }}^{(2)}, \ldots, c_{\text {sup }}^{(N)}\right),
$$

respectively, and the $m N \times m N$ triangular matrix

$$
\tilde{G}_{\text {sup }}:=\left(\begin{array}{cccc}
G_{\text {sup }}^{1} & 0_{m \times m} & \ldots 0_{m \times m} & 0_{m \times m} \\
F_{1 \text { sup }}^{2} & G_{\text {sup }}^{2} & \ldots 0_{m \times m} & 0_{m \times m} \\
\ldots & \ldots & \ldots & \ldots \\
F_{1 \text { sup }}^{N-1} & F_{2 s u p}^{N-1} & \ldots G_{\text {sup }}^{N-1} & 0_{m \times m} \\
F_{1 \text { sup }}^{N} & F_{2 s u p}^{N} & \ldots F_{\text {sup }}^{N} & G_{\text {sup }}^{N}
\end{array}\right) .
$$

Then the algebraic equations (12) can be rewritten in the form

$$
\hat{\nu}^{T} \tilde{G}_{\text {sup }}-c_{\text {sup }}=0,
$$

Copyright $@$ ㅇ by SIAM. Unauthorized reproduction of this article is prohibited. 
where (Definition 2) this matrix is nonsingular and therefore $\hat{\nu}^{T}=c_{\text {sup }} \tilde{G}_{\text {sup }}^{-1}$. Note also that the matrix $\tilde{G}_{\text {sup }}$ can be written in block form as

$$
\tilde{G}_{\text {sup }}=\left(\begin{array}{c|c|c|c} 
& & 0_{m \times m} \\
& 0_{m \times m} \\
g_{k 1}(t), t \in \tau_{\text {sup }}^{1} & g_{k 2}(t), t \in \tau_{\text {sup }}^{2} & \ldots & \vdots \\
k=1,2, \ldots, N & k=2,3, \ldots, N & 0_{m \times m} \\
& & g_{k N}(t), t \in \tau_{\text {sup }}^{N} \\
k=N
\end{array}\right),
$$

where the nonzero block entries are given by

$$
\left(\begin{array}{cc}
g_{k j}(t), & t \in \tau_{\text {sup }}^{j} \\
j \leq k \leq N, & j=1, \ldots, N
\end{array}\right)
$$

To establish the new optimality conditions, define the so-called cocontrol $1 \times N$ vector function

$$
\Delta(t)=\left(\Delta_{1}(t), \ldots, \Delta_{N}(t)\right)
$$

as

$$
\begin{aligned}
\Delta_{1}(t)= & \nu^{(1) T} g_{11}(t)+\nu^{(2) T} g_{21}(t)+\cdots+\nu^{(N) T} g_{N 1}(t)-c_{1}(t), \\
& \ldots \ldots \ldots \ldots \ldots \ldots \ldots \ldots \ldots \ldots \\
\Delta_{N-1}(t)= & \nu^{(N-1) T} g_{N-1 N-1}(t)+\nu^{(N) T} g_{N N-1}(t)-c_{N-1}(t), \\
\Delta_{N}(t)= & \nu^{(N) T} g_{N N}(t)-c_{N}(t),
\end{aligned}
$$

or, on introducing the $1 \times N$ vector function,

$$
\begin{aligned}
c(t) & =\left(c_{1}(t), \ldots, c_{N}(t)\right), \\
\Delta(t) & =\hat{\nu}^{T} \hat{G}(t)-c(t),
\end{aligned}
$$

where $\hat{G}(t)$ is an $m N \times N$ matrix of the form

$$
\hat{G}(t)=\left(\begin{array}{cccc}
g_{11}(t) & 0_{m \times 1} & \ldots & 0_{m \times 1} \\
g_{21}(t) & g_{22}(t) & \ldots & 0_{m \times 1} \\
\ldots & \ldots & \ldots & \ldots \\
g_{N 1}(t) & g_{N 2}(t) & \ldots & g_{N N}(t)
\end{array}\right) .
$$

Note also that the $m N \times m N$ matrix $\tilde{G}_{\text {sup }}$ is obtained from $\hat{G}(t)$ in an obvious manner by evaluating the rows of the matrix $\hat{G}(t)$ at the supporting moments $t \in \tau_{\text {sup }}^{k}, k=$ $1, \ldots, N$.

DEFINITION 4. We say that the supporting control function $\left\{\tau_{\text {sup }}^{k}, u_{k}(t), k=\right.$ $1, \ldots, N\}$ is nondegenerate for the problem (1)-(3) if

$$
\frac{d \Delta_{k}\left(\tau_{j}\right)}{d t} \neq 0 \quad \forall \tau_{j} \in \tau_{\text {sup }}^{k}, \quad k=1, \ldots, N .
$$

Remark 2. Here nondegeneracy means that in a small neighborhood of the supporting points the admissible control can be replaced by constant functions whose 
values are less than those on the control constraint boundary and satisfy (11); i.e., the support control function is nonsingular if there exist numbers $\lambda_{0}>0, \mu_{0}>$ $0, u_{j}^{k}(\lambda), j=1, \ldots, m, k=1, \ldots, N$, such that the following equalities:

$$
\begin{array}{r}
\sum_{j=1}^{k} \sum_{i=1}^{m} u_{j}^{i}(\lambda) \int_{\tau_{i j}-\lambda}^{\tau_{i j}+\lambda} g_{k j}(t) d t=\sum_{j=1}^{k} \sum_{i=1}^{m} \int_{\tau_{i j}-\lambda}^{\tau_{i j}+\lambda} g_{k j}(t) u_{j}(t) d t, \\
\left|u_{j}^{k}\right| \leq 1-\mu_{0}, \quad j=1, \ldots, m, \quad k=1, \ldots, N,
\end{array}
$$

hold for all $\lambda, 0<\lambda<\lambda_{0}$, and $k, 1 \leq k \leq N$. This fact will be used below in the proof of the optimality conditions.

Associate with each supporting time instance $\tau_{k j}$ a small subinterval $T_{k j}$ from $T$ such that the matrix $G_{g e n}^{k}:=\left\{\int_{T_{k j}} g_{k k}(\tau) d \tau, j=1, \ldots, m\right\}$ is nonsingular. Also without loss of generality we can assume that $\tau_{k j}$ is one or the other of the end points of $T_{k j}$ and the supporting control functions $u_{k}(t)=u_{j}^{k}$ for $t \in T_{k j}, j=1, \ldots, N$, are constant over the segments $T_{k j}$. Then we have the following result.

THEOREM 1. A supporting control function $\left\{\tau_{\text {sup }}^{k}, u_{k}^{0}(t), k=1, \ldots, N\right\}$ is an optimal solution of the problem (1)-(4) if

$$
u_{k}^{0}(t)=-\operatorname{sgn}\left(\Delta_{k}(t)\right), \quad k=1, \ldots, N, t \in T .
$$

Moreover, if this supporting control function is nondegenerate, then the above condition is necessary and sufficient.

Proof. Let $u_{k}(t) \neq u_{k}^{0}(t), k=1, \ldots, N$, be an admissible control and $x_{k}(t)$ the corresponding trajectory of the system (1)-(2). Then standard transformations yield that the increment $\Delta J(u):=J\left(u^{0}\right)-J(u)$ of the cost function can be expressed in the form

$$
\Delta J(u)=\int_{0}^{t^{*}} \sum_{j=1}^{N} c_{j}(t)\left[u_{j}^{0}(t)-u_{j}(t)\right] d t=-\sum_{j=1}^{N} \int_{0}^{t *} \Delta_{j}(t)\left[u_{j}^{0}(t)-u_{j}(t)\right] d t .
$$

Hence by using (18) we have that $\Delta J(u) \geq 0$ for any admissible control $u$; i.e., $\left\{\tau_{\text {sup }}^{k}, u_{k}^{0}\right\}$ is an optimal supporting control function.

Let $\left\{\tau_{\text {sup }}^{k}, u_{k}^{0}(t), k=1, \ldots, N\right\}$ be an optimal nondegenerate control, but there exists $k_{*}, 1 \leq k_{*} \leq N$, and there exists $t_{*} \in T$ such that the theorem is not valid. Suppose also that $t_{*} \in\left[\tau_{k_{*} j}-\lambda, \tau_{k_{*} j}+\lambda\right]$, where $\lambda>0$ is a small number; i.e., the instance $t_{*}$ lies in an neighborhood of some supporting time instance $\tau_{k_{*} j}$. Then since the supporting control is nondegenerate there exists a control variation $\Delta u_{k_{*}}^{0}(t)$, defined on the intervals $\left[\tau_{k_{*} j}-\lambda, \tau_{k_{*} j}+\lambda\right]$, such that $J\left(u^{0}\right)>0$, which contradicts the optimality of $u_{k}^{0}(t)$.

Given this last fact, we now suppose that $t_{*} \notin\left[\tau_{k_{*} j}-\lambda, \tau_{k_{*} j}+\lambda\right]$ for all $j=$ $1, \ldots, m$, for some small $\lambda>0$. Also, without loss of generality, we assume that $\Delta_{k_{*}}^{0}\left(t_{*}\right)>0$ and $u_{k_{*}}\left(t_{*}\right)>0$. Then, by continuity of $\Delta_{k_{*}}(t)$ and piecewise-continuity of $u_{k_{*}}(t)$, there exists a neighborhood $T_{k_{*}}\left(t_{*}\right)$ of $t_{*}$, such that $\Delta_{k_{*}}(t)>0, u_{k_{*}}(t)>-1$ for $t \in T_{k_{*}}\left(t_{*}\right)$. Now we have to construct the admissible control variation such that the corresponding increment of the cost function satisfies $\Delta J(u)>0$, which is impossible for the optimal controls $u_{k}^{0}(t)$.

Consider now the case of a small real number $\lambda_{0}>0$ (we see below that the existence of such a number $\lambda_{0}$ is guaranteed by the fact that the supporting control is nondegenerate), and for all $\lambda, 0<\lambda<\lambda_{0}$, define the control variation $\Delta u(t)=$ 
$\left(\Delta u_{1}(t), \ldots, \Delta u_{N}(t)\right), t \in T$, as

$$
\begin{aligned}
\Delta u_{k}(t) & =0, \quad k<k_{*}, t \in T, \\
\Delta u_{k_{*}}(t) & =\left\{\begin{array}{l}
\theta\left(-1-u_{k_{*}}(t)\right), \quad \theta>0, \quad t \in T_{k_{*}}(t), \\
0, \quad t \in T \backslash\left(\bigcup_{j=1}^{m}\left[\tau_{k_{*} j}-\lambda, \tau_{k_{*} j}+\lambda\right] \cup T_{k_{*}}(t)\right) .
\end{array}\right.
\end{aligned}
$$

We now have that the control variations on the intervals $\left[\tau_{k_{*} j}-\lambda, \tau_{k_{*} j}-\lambda\right], j=$ $1, \ldots, m$, can be chosen as constant functions $\Delta u_{k_{*}}(t) \equiv \Delta \vartheta_{j}^{k}(\lambda)$. Those for the remaining passes $k>k_{*}$ are defined as

$$
\begin{gathered}
\Delta u_{k}(t) \equiv 0, \quad k=k_{*}+1, \ldots, N, \quad t \in T \backslash \bigcup_{j=1}^{m}\left[\tau_{k j}-\lambda, \tau_{k j}+\lambda\right], \\
\Delta u_{k}(t) \equiv \Delta \vartheta_{j}^{k}(\lambda), t \in\left[\tau_{k j}-\lambda, \tau_{k j}+\lambda\right], \quad j=1, \ldots, m, \quad k>k_{*},
\end{gathered}
$$

where the $\Delta \vartheta_{j}^{k}(\lambda)$ are unknown constants determined below.

By using (11), it follows that the conditions

$$
\int_{0}^{\alpha} \sum_{s=1}^{k} g_{k s}(\tau) \Delta u_{s}(\tau) d \tau=0, \quad k=1, \ldots, N,
$$

hold for any admissible variation $\Delta u(t)$, and these can be rewritten in the form

$$
\begin{aligned}
& \phi_{k_{*}}(\lambda)=\sum_{j=1}^{m} \int_{\tau_{k_{*} j}-\lambda}^{\tau_{k_{*} j}+\lambda} g_{k_{*} k_{*}}(\tau) \vartheta_{j}^{k_{*}}(\lambda) d \tau \\
& =-\theta \int_{T_{k_{*}\left(t_{*}\right)}} g_{k_{*} k_{*}}(\tau)\left(-1-u_{k_{*}}(\tau)\right) d \tau, \\
& \phi_{k_{*}+1}(\lambda):=\sum_{j=1}^{m} \int_{\tau_{k_{*}+1 j}-\lambda}^{\tau_{k_{*}+1 j}+\lambda} g_{k_{*}+1 k_{*}+1}(\tau) \vartheta_{j}^{k_{*}+1}(\lambda) d \tau \\
& =-\sum_{j=1}^{m} \int_{\tau_{k_{*} j}-\lambda}^{\tau_{k_{*} j}+\lambda} g_{k_{*}+1 k_{*}}(\tau) \vartheta_{j}^{k_{*}}(\lambda) d \tau-\theta \int_{T_{k_{*}}\left(t_{*}\right)} g_{k_{*}+1 k_{*}}(\tau)\left(-1-u_{k_{*}}(\tau)\right) d \tau,
\end{aligned}
$$

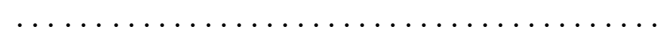

$$
\begin{aligned}
& \phi_{N}(\lambda)=\sum_{j=1}^{m} \int_{\tau_{N j-\lambda}}^{\tau_{N j+\lambda}} g_{N N}(\tau) \vartheta_{j}^{N}(\lambda) d \tau-\sum_{j=1}^{m} \int_{\tau_{k_{* j}-\lambda}}^{\tau_{k_{* j}+\lambda}+\lambda} g_{N k_{*}}(\tau) \vartheta_{j}^{k_{*}}(\lambda) d \tau \\
& -\theta \int_{T_{k_{*}}\left(t_{*}\right)} g_{N k_{*}}(\tau)\left(-1-u_{k_{*}}(\tau)\right) d \tau \\
& -\cdots-\sum_{j=1}^{m} \int_{\tau_{N-1 j}-\lambda}^{\tau_{N-1 j}+\lambda} g_{N N-1}(\tau) \vartheta_{j}^{N-1}(\lambda) d \tau .
\end{aligned}
$$

Expanding the function $\phi_{k_{*}}(\lambda)$ of (20) as a Taylor series and setting $\Delta \vartheta_{\lambda}^{k_{*}}=$ $\left(\Delta \vartheta_{1}^{k_{*}}(\lambda), \ldots, \Delta \vartheta_{m}^{k_{*}}(\lambda)\right)$ now yields

$$
\begin{aligned}
2 \lambda G_{\text {sup }}^{k_{*}} \Delta \vartheta_{\lambda}^{k_{*}} & +\frac{\lambda^{3}}{3}\left\{\frac{d^{2} g_{k_{*} k_{*}}\left(\tau_{k_{*} j}\right)}{d \tau}, j=1, \ldots, m\right\} \Delta \vartheta_{\lambda}^{k_{*}}+o_{k_{*}}\left(\lambda^{3}\right) \\
& =-\theta \int_{T_{k_{*}}\left(t_{*}\right)} g_{k_{*} k_{*}}(\tau)\left(-1-u_{k_{*}}(\tau)\right) d \tau,
\end{aligned}
$$

Copyright $@$ by SIAM. Unauthorized reproduction of this article is prohibited. 
where $o_{k_{*}}\left(\lambda^{3}\right)$ denotes terms of degree 3 and above, which are neglected here. Hence the required vector $\Delta \vartheta_{\lambda}^{k_{*}}$ can be represented as

$\Delta \vartheta_{\lambda}^{k_{*}}=\frac{1}{\lambda} \theta \hat{u}_{k_{*}}+\theta o_{k_{*}}(\lambda), \quad$ where $\quad \hat{u}_{k_{*}}=-\frac{1}{2} G_{s u p}^{k_{*}^{-1}} \int_{T_{k_{*}}\left(t_{*}\right)} g_{k_{*} k_{*}}(\tau)\left(-1-u_{k_{*}}(\tau)\right) d \tau$,

and $o_{k_{*}}(\lambda)$ denotes a residual first order term. Using (17) and (21), it follows that for a small value of $\lambda \in\left(0, \lambda_{0}\right)$ there exists the real number $\theta=\theta(\lambda)$ such that $\theta(\lambda)=\mu_{k_{*}} \lambda \leq 1$, where $\mu_{k_{*}}>0$ does not depend on $\lambda$, and the following inequalities:

$$
\left|u_{j}^{k_{*}}(\lambda)+\Delta \vartheta_{j}^{k_{*}}(\lambda)\right| \leq 1, \quad j=1, \ldots, m,
$$

hold. Here we have exploited the fact that the admissible controls are constants $u_{j}^{k}(\lambda)$ over the intervals $T_{j}^{k}$, containing the supporting points $\tau_{k j}$. Hence, the function

$$
\bar{u}_{k_{*}}(t)=\left\{\begin{array}{l}
u_{j}^{k_{*}}(\lambda)+\Delta \vartheta_{j}^{k_{*}}(\lambda), t \in\left[\tau_{k_{*} j}-\lambda, \tau_{k_{*} j}+\lambda\right] \\
u_{k_{*}}(t)+\theta(\lambda)\left(-1-u_{k_{*}}(t)\right), \quad t \in T_{k_{*}}\left(t_{*}\right)
\end{array}\right.
$$

is an admissible control function for $\theta(\lambda)=\mu_{k_{*}} \lambda \leq 1$ and a sufficiently small $\mu_{k_{*}}$.

In order to find $\Delta \vartheta_{\lambda}^{k_{*}+1}$ and $\theta(\lambda)$, expand $\phi_{k_{*}+1}(\lambda)$ as a Taylor series to yield

$$
\begin{aligned}
\sum_{j=1}^{m} \int_{\tau_{k_{*}-\lambda}-\lambda}^{\tau_{k_{*}}+\lambda} g_{k_{*}+1 k_{*}}(\tau) \Delta \vartheta_{j}^{k_{*}}(\lambda) d \tau & =2 \lambda \sum_{j=1}^{m} g_{k_{*}+1 k_{*}}\left(\xi_{j}\right) \Delta \vartheta_{j}^{k_{*}}(\lambda) \\
& =2 \lambda \tilde{G}_{\xi}^{k_{*}+1} \Delta \vartheta_{\lambda}^{k_{*}+1} \\
& =2 \lambda \tilde{G}_{\xi}^{k_{*}+1}\left(\frac{1}{\lambda} \mu_{k_{*}} \lambda \hat{u}_{k_{*}}+\mu_{k_{*}} \lambda o_{k_{*}}(\lambda)\right) \\
& =2 \tilde{G}_{\xi}^{k_{*}+1} \mu_{k_{*}} \lambda \hat{u}_{k_{*}}+\mu_{k_{*}} \breve{o}_{k_{*}}\left(\lambda^{3}\right) .
\end{aligned}
$$

Here the matrix $\tilde{G}_{\xi}^{k_{*}+1}$ is constructed from the rows $\left\{g_{k_{*}+1 k_{*}}\left(\xi_{j}\right), j=1, \ldots, m\right\}$, where $\xi_{j}$ are points from the intervals $\left[\tau_{k_{*} j}-\lambda, \tau_{k_{*} j}+\lambda\right]$. Next, set $\Delta \vartheta_{\lambda}^{k_{*}+1}=$ $\left(\Delta \vartheta_{1}^{k_{*}+1}(\lambda), \ldots, \Delta \vartheta_{m}^{k_{*}+1}(\lambda)\right)$ to obtain

$$
\begin{aligned}
2 \lambda G_{\text {sup }}^{k_{*}+1} \Delta \vartheta_{\lambda}^{k_{*}+1}+ & \frac{\lambda^{3}}{3}\left\{\frac{d^{2} g_{k_{*}+1 k_{*}+1}\left(\tau_{k_{*} j}\right)}{d \tau}, j=1, \ldots, m\right\} \Delta \vartheta_{\lambda}^{k_{*}+1}+o_{k^{*}+1}\left(\lambda^{3}\right) \\
= & -\mu_{k_{*}} \lambda\left\{\tilde{G}_{\xi}^{k_{*}+1} \hat{u}_{k_{*}}+\int_{T_{k_{*}}\left(t_{*}\right)} g_{k_{*}+1 k_{*}+1}(\tau)\left(-1-u_{k_{*}}(\tau)\right) d \tau\right\} \\
& +\mu_{k_{*}} \breve{o}_{k_{*}}\left(\lambda^{3}\right),
\end{aligned}
$$

and hence the required vector $\Delta \vartheta_{\lambda}^{k_{*}+1}$ can be expressed as

$$
\begin{aligned}
\Delta \vartheta_{\lambda}^{k_{*}+1} & =\frac{1}{\lambda} \mu_{k_{*}} \lambda \hat{u}_{k_{*}+1}+\mu_{k_{*}} \lambda o_{k^{*}+1}(\lambda), \\
\hat{u}_{k_{*}+1} & =-\frac{1}{2}\left(G_{\text {sup }}^{k_{*}+1}\right)^{-1}\left\{\tilde{G}_{\xi}^{k_{*}+1} \hat{u}_{k_{*}}-\int_{T_{k_{*}}\left(t_{*}\right)} g_{k_{*}+1 k_{*}+1}(\tau)\left(-1-u_{k_{*}}(\tau)\right) d \tau\right\} .
\end{aligned}
$$

Now choose $\Delta \vartheta_{\lambda}^{k_{*}+1}$ such that the following inequalities hold:

$$
\left|u_{j}^{k_{*}+1}(\lambda)+\Delta \vartheta_{j}^{k_{*}+1}(\lambda)\right| \leq 1, \quad j=1, \ldots, m,
$$

Copyright $@$ by SIAM. Unauthorized reproduction of this article is prohibited. 
and hence the values of $\mu_{k_{*}}$ and $\lambda_{0}$ can be decreased as required. Continuing this expansion procedure for the remaining equations in (20), we obtain the desired admissible control function in the form

$$
\bar{u}(t)=u^{0}(t)+\Delta u(t)=\left\{u_{1}^{0}(t)+\Delta u_{1}(t), \ldots, u_{N}^{0}(t)+\Delta u_{N}(t)\right\}, \quad t \in T,
$$

and note here that $\Delta u_{k}(t)=0$ for all $k<k_{*}$.

At this stage we can calculate the increment of the cost function generated by the designed control function $\bar{u}(t)$ as

$$
\begin{aligned}
\Delta J(u)= & J(\bar{u})-J\left(u^{0}\right)=\sum_{k=1}^{N} \int_{0}^{\alpha} \Delta_{k}(t) \Delta u_{k}(t) d t=\sum_{k=k_{*}}^{N} \int_{0}^{\alpha} \Delta_{k}(t) \Delta u_{k}(t) d t \\
= & -\theta \int_{T_{k_{*}}\left(t_{*}\right)} \Delta_{k_{*}}(t)\left(-1-u_{k_{*}}(t)\right) d t \\
& -\sum_{j=1}^{m} \int_{\tau_{k_{*} j}-\lambda}^{\tau_{k_{*} j}+\lambda} \Delta_{k_{*}}(t)\left[u_{j}^{k_{*}}(\lambda)+\Delta \vartheta_{j}^{k_{*}}(\lambda)-u_{j}^{k_{*}}(t)\right] d t \\
& -\sum_{s=k_{*}+1}^{N} \sum_{j=1}^{m} \int_{\tau_{s j}-\lambda}^{\tau_{s j}+\lambda} \Delta_{s}(t)\left[u_{j}^{s}(\lambda)+\Delta \vartheta_{j}^{s}(\lambda)-u_{j}^{s}(t)\right] d t .
\end{aligned}
$$

Since $\Delta_{k}\left(\tau_{k j}\right)=0, k=k_{*}, \ldots, N, j=1, \ldots, m$, then, again using the Taylor series expansion in $\lambda$, we have the following estimate for the integral components:

$$
\begin{aligned}
\int_{\tau_{s j}-\lambda}^{\tau_{s j}+\lambda} \Delta_{s}(t)\left[u_{j}^{s}(\lambda)+\Delta \vartheta_{j}^{s}(\lambda)-u_{j}^{s}(t)\right] d t= & \int_{\tau_{s j}}^{\tau_{s j}} \Delta_{s}(t)\left[u_{j}^{s}(\lambda)+\Delta \vartheta_{j}^{s}(\lambda)-u_{j}^{s}(t)\right] d t \\
& +2 \lambda \Delta_{s}\left(\tau_{s j}\right)\left[u_{j}^{s}(\lambda)+\Delta \vartheta_{j}^{s}(\lambda)-u_{j}^{s}\left(\tau_{s j}\right)\right] \\
& +\lambda^{2} \frac{d \Delta_{s}\left(\tau_{s j}\right)}{d t}\left[u_{j}^{s}(\lambda)+\Delta \vartheta_{j}^{s}(\lambda)-u_{j}^{s}\left(\tau_{s j}\right)\right] \\
& +o_{1}\left(\lambda^{2}\right) \cong o\left(\lambda^{2}\right) .
\end{aligned}
$$

Hence (25) and (26) yield

$$
\Delta J(u)=-\mu_{k_{*}} \lambda \int_{T_{k_{*}}\left(t_{*}\right)} \Delta_{k_{*}}(t)\left(-1-u_{k_{*}}(t)\right) d t+o(\lambda)>0
$$

for a sufficiently small $\lambda>0$, which contradicts the optimality of control functions $u_{k}^{0}(t), k=1, \ldots, N$.

Remark 3. The analysis which now follows shows that the above result can be reformulated in the traditional maximum principle form. In particular, it will be shown that the cocontrol functions $\Delta_{k}(t), t \in T$, here are connected directly to the adjoint (dual) variables $\psi_{k}(t), t \in T$, as $\Delta_{k}(t)=-\psi_{k}^{T}(t) b$. Note also that the term $\psi_{k}^{T}(t) b$ is part of the Hamiltonian function which arises in the maximum principle statement of the result here. Moreover, the vectors $\left\{\nu^{(k)}, k=1, \ldots, N\right\}$ (termed Lagrange multipliers in some literature) will be used as the boundary conditions for the corresponding differential equations describing the adjoint (dual) variables $\psi_{k}(t)$ (in contrast to the classic maximum principle, where such boundary conditions are not specified).

Copyright $@$ by SIAM. Unauthorized reproduction of this article is prohibited. 
Let $\psi_{N}(t)$ be the solution of

$$
\frac{d \psi_{N}(t)}{d t}=-A^{T} \psi_{N}(t), \quad \psi_{N}(\alpha)=p_{N}-H_{N}^{T} \nu^{N}, \quad t \in T,
$$

or

$$
\psi_{N}(t)=K_{1}^{T}(\alpha-t) \psi(\alpha), \quad t \in T
$$

Hence

$$
\begin{aligned}
\psi_{N}^{T}(t) b= & \left(p_{N}^{T}-\left(\nu^{N}\right)^{T} H_{N}\right) K_{1}(\alpha-t) b=p_{N}^{T} K_{1}(\alpha-t) b \\
& -\left(\nu^{N}\right)^{T} H_{N} K_{1}(\alpha-t) b=c_{N}(t)-\left(\nu^{N}\right)^{T} g_{N N}(t)=-\Delta_{N}(t) .
\end{aligned}
$$

In order to verify the validity of the corresponding conditions for subsequent passes we use (9) for the differential equations (7). Let $\psi_{N-1}(t), t \in T$, be a solution of the differential equation

$$
\frac{d \psi_{N-1}(t)}{d t}=-A^{T} \psi_{N-1}(t)-D^{T} \psi_{N}(t), \quad \psi_{N-1}(\alpha)=p_{N-1}-H_{N-1}^{T} \nu^{N-1}, t \in T .
$$

Then

$$
\begin{aligned}
\psi_{N-1}^{T}(t) b= & \left(p_{N-1}^{T}-\left(\nu^{N-1}\right)^{T} H_{N-1}\right) K_{1}(\alpha-t) b \\
& -\left(p_{N}^{T}-\left(\nu^{N}\right)^{T} H_{N}\right) \int_{0}^{t} K_{1}^{T}(t-\tau) D^{T} K_{1}^{T}(\alpha-\tau) b d \tau \\
= & p_{N-1}^{T} K_{1}(\alpha-t) b-\left(\nu^{N-1}\right)^{T} H_{N-1} K_{1}(\alpha-t) b \\
& -\left(p_{N}^{T}-\left(\nu^{N}\right)^{T} H_{N}\right) K_{2}(\alpha-t) b \\
= & c_{N-1}(t)-\left(\nu^{N-1}\right)^{T} g_{N-1 N-1}(t)-\left(\nu^{N}\right)^{T} g_{N N-1}(t)=-\Delta_{N-1}(t) .
\end{aligned}
$$

By analogy with the case for (31)-(32), we have

$$
\psi_{k}^{T}(t) b=-\Delta_{k}(t), \quad k=2, \ldots, N,
$$

where $\psi_{k}(t), t \in T$, are the solutions of the following differential equations:

$$
\frac{d \psi_{k}(t)}{d t}=-A^{T} \psi_{k}(t)-D^{T} \psi_{k+1}(t), \quad \psi_{k}(\alpha)=p_{k}-H_{k}^{T} \nu^{k}, \quad t \in T .
$$

For each $k=1, \ldots, N$ introduce the associated Hamilton function as

$$
H_{k}\left(x_{k-1}, x_{k}, \psi_{k}, u_{k}\right)=\psi_{k}^{T}\left(A x_{k}+D x_{k-1}+b u_{k}\right), \quad t \in T .
$$

Then the use of (33) yields that the optimality conditions (18) can be reformulated in maximum principle form as the following corollary to Theorem 1 .

COROLlary 1. The admissible supporting control $\left\{\tau_{\text {sup }}^{k}, u_{k}^{0}(t), k=1, \ldots, N\right\}$ is optimal if along the corresponding trajectories $x_{k}^{0}(t), \psi_{k}(t)$ of (1)-(2) and (34) the Hamiltonian function has maximum value, i.e.,

$$
H_{k}\left(x_{k-1}^{0}(t), x_{k}^{0}(t), \psi_{k}, u_{k}^{0}(t)\right)=\max _{|v| \leq 1} H_{k}\left(x_{k-1}^{0}(t), x_{k}^{0}(t), \psi_{k}, v\right), \quad t \in T,
$$

Copyright $@$ by SIAM. Unauthorized reproduction of this article is prohibited. 
for $k=1, \ldots, N$. If the admissible supporting control is nondegenerate, then this condition is necessary and sufficient.

Remark 4. In order to further emphasize the relationship between the support elements and the control function, note that the optimality conditions given by Theorem 1 can be equivalently stated in the form

$$
\begin{aligned}
& \Delta_{k}(t)>0 \text { at } u_{k}^{0}(t)=-1, \quad \Delta_{k}(t)<0 \text { at } u_{k}^{0}(t)=1, \\
& \Delta_{k}(t)=0 \text { at }-1<u_{k}^{0}(t)<1, \quad k=1,2, \ldots, N, t \in T .
\end{aligned}
$$

Hence the supporting elements and control function of optimal solution are interconnected such that the supporting instances are the switching moments for optimal bang-bang control functions.

In the next section, the maximum principle for arbitrary admissible control functions of (1)-(4) is established using the suboptimality conditions.

3.1. $\epsilon$-optimality conditions. Often in the numerical implementation of optimal control algorithms it is beneficial to exploit approximate solutions with corresponding error estimation. Hence it is necessary to introduce the "suboptimality" concept as it is often sufficient to stop the numerical computations when a satisfactory accuracy level has been achieved.

Assume that $\left\{u_{k}^{0}(t), k \in K\right\}$ is the optimal control for (1)-(4), and let $J\left(u^{0}\right)$ denote the corresponding optimal cost function value.

Definition 5. We say that the admissible control function $\left\{u_{k}^{\epsilon}(t), k \in K\right\}$ is $\epsilon$-optimal if the corresponding solution $\left\{x_{k}^{\epsilon}(t), t \in T, k \in K\right\}$ of (1)-(4) satisfies $J\left(u^{0}\right)-J\left(u^{\epsilon}\right) \leq \epsilon$.

Now we proceed to calculate an estimate of a supporting control function

$$
\left\{u_{k}, \tau_{\text {sup }}^{k}, k \in K, t \in T\right\},
$$

i.e., a measure of the nonoptimality of the control. Note also that this estimate can be partitioned into two principal parts: one of which evaluates the degree of nonoptimality of the chosen admissible control functions $u_{k}(t)$, and the second the error produced by nonoptimality of the support $\tau_{\text {sup }}^{k}$. This partition is a major advantage in the design of numerically applicable solution algorithms.

Introduce an estimate of optimality $\beta=\beta\left(\tau_{\text {sup }}, u\right)$ as the value of the maximum increment for the cost function here calculated in the absence of the principal constraints (4); i.e., this estimate is given by the solution of the following relaxed optimization problem:

$$
\max _{\Delta u_{k}} \Delta J(u),\left|u_{k}(t)+\Delta_{k} u(t)\right| \leq 1, t \in T, k=1, \ldots, N
$$

It is easy to see that

$$
\beta=\beta\left(\tau_{\text {sup }}, u\right)=\sum_{k=1}^{N} \int_{T_{k}^{+}} \Delta_{k}(t)\left(u_{k}(t)+1\right) d t+\sum_{k=1}^{N} \int_{T_{k}^{-}} \Delta_{k}(t)\left(u_{k}(t)-1\right) d t
$$

where

$$
T_{k}^{+}=\left\{t \in T: \Delta_{k}(t)>0\right\}, \quad T_{k}^{-}=\left\{t \in T: \Delta_{k}(t)<0\right\},
$$

and we have the following result. 
Theorem 2 ( $\epsilon$-maximum principle). Given any $\epsilon \geq 0$, the admissible control $\left\{u_{k}(t), t \in T, k \in K\right\}$ is $\epsilon$-optimal for (1)-(4) if and only if there exists a support $\left\{\tau_{\text {sup }}^{k}, k \in K\right\}$ such that along the solutions $x_{k}(t), \psi_{k}(t), t \in T, k \in K$, of (1)-(4) and (34) the Hamiltonian attains its $\epsilon$-maximum value, i.e.,

$$
H_{k}\left(x_{k-1}(t), x_{k}(t), \psi_{k}, u_{k}(t)\right)=\max _{|v| \leq 1} H_{k}\left(x_{k-1}(t), x_{k}(t), \psi_{k}, v\right)-\epsilon_{k}(t), \quad t \in T,
$$

where the functions $\epsilon_{k}(t), k \in K$, satisfy the following inequality:

$$
\sum_{k \in K} \int_{T} \epsilon_{k}(t) d t \leq \epsilon
$$

Proof. Assume that (40) and (41) hold for an admissible control $\left\{u_{k}(t), t \in\right.$ $T, k \in K\}$. Then by (33) the suboptimal estimate is

$$
\begin{aligned}
\beta= & \beta\left(\tau_{\text {sup }}, u\right)=\sum_{k=1}^{N} \int_{T_{k}^{+}} \psi_{k}^{T}(t) b\left(-u_{k}(t)-1\right) d t+\sum_{k=1}^{N} \int_{T_{k}^{-}} \psi_{k}^{T}(t) b\left(1-u_{k}(t)\right) d t \\
= & \sum_{k=1}^{N} \int_{T_{k}^{+}} \psi_{k}^{T}(t)\left(A x_{k}(t)+D x_{k-1}(t)-b\right) d t \\
& -\sum_{k=1}^{N} \int_{T_{k}^{+}} \psi_{k}^{T}(t)\left(A x_{k}(t)+D x_{k-1}(t)+b u_{k}(t)\right) d t \\
= & \sum_{k=1}^{N} \int_{T_{k}^{-}} \psi_{k}^{T}(t)\left(A x_{k}(t)+D x_{k-1}(t)+b\right) d t \\
& -\sum_{k=1}^{N} \int_{T_{k}^{-}} \psi_{k}^{T}(t)\left(A x_{k}(t)+D x_{k-1}(t)-b u_{k}(t)\right) d t \\
& +\sum_{k=1}^{N} \int_{T}\left[\max _{|v| \leq 1} H_{k}\left(x_{k-1}(t), x_{k}(t), \psi_{k}(t), v\right)-H_{k}\left(x_{k-1}(t), x_{k}(t), \psi_{k}(t), u_{k}(t)\right)\right] d t \\
= & \sum_{k=1}^{N} \int_{T} \epsilon_{k}(t) d t \leq \epsilon .
\end{aligned}
$$

Since the suboptimal estimate (38) has been calculated in the absence of constraints (4), then it is obvious that

$$
J\left(u^{0}\right)-J(u) \leq \beta\left(\tau_{\text {sup }}, u\right) \leq \epsilon .
$$

This proves the $\epsilon$-optimality property of the admissible control $\left\{u_{k}(t), t \in T, k \in K\right\}$.

For the converse argument, let $\left\{u_{k}(t), t \in T, k \in K\right\}$ be an $\epsilon$-optimal admissible control, and let $\left\{\tau_{\text {sup }}^{k}, k \in K\right\}$ be an arbitrary support. Then the suboptimal estimate of the control corresponding to the chosen support is given by

$$
\beta\left(\tau_{\text {sup }}, u\right)=\sum_{k=1}^{N} \int_{T} \Delta_{k}(t) u_{k}(t) d t+\sum_{k=1}^{N} \int_{T_{k}^{+}} \Delta_{k}(t) d t-\sum_{k=1}^{N} \int_{T_{k}^{-}} \Delta_{k}(t) d t .
$$

Copyright $@$ ㅇ by SIAM. Unauthorized reproduction of this article is prohibited. 
Also introduce the following dual optimization problem:

$$
\min _{y, v, w} I(y, v, w), \quad I(y, v, w)=\sum_{k \in K}\left[h_{k}^{T} y_{k}+\int_{T} v_{k}(t) d t+\int_{T} w_{k}(t) d t\right],
$$

subject to

$$
\sum_{s=k}^{N} y_{s}^{T} g_{s k}(t)-v_{k}(t)+w_{k}(t)=c_{k}(t), \quad v_{k}(t) \geq 0, w_{k}(t) \geq 0, t \in T, k \in K .
$$

At this stage we have to check that this dual optimization problem has a nonempty set of admissible variables $z_{k}=\left\{y_{k}, v_{k}, w_{k}, k \in K\right\}$. Suppose therefore that we denote the chosen support by $\tau_{\text {sup }}^{k}, k \in K$, and then use (18) to construct the vectors $z_{k}=$ $\left\{y_{k}, v_{k}, w_{k}, k \in K\right\}$ as

$$
\begin{aligned}
y_{k} & =\nu_{k}, \nu_{k}(t)=\Delta_{k}(t) ; w_{k}(t)=0 \text { if } \Delta_{k}(t) \geq 0, \\
v_{k}(t) & =0, w_{k}(t)=\Delta_{k}(t) \text { if } \Delta_{k}(t)<0 .
\end{aligned}
$$

Then, by (18), these satisfy the constraint (44) of the dual problem. Also since this dual problem has a nonempty set of feasible variables

$$
\left\{y_{k}, v_{k}, w_{k}, k \in K\right\}
$$

it is routine to show that it has an optimal solution if there exists an optimal control for (1)-(4).

Let $\left\{y_{k}^{0}, v_{k}^{0}(t), w_{k}^{0}(t), t \in T, k \in K\right\}$ denote an optimal solution of (43)-(44). Then (43) and (18) yield

$$
\begin{aligned}
\beta\left(\tau_{\text {sup }}, u\right)= & \sum_{k=1}^{N} \sum_{s=k}^{N} \int_{T} \nu_{s}^{T}(t) g_{s k}(t) u_{k}(t) d t-\sum_{k=1}^{N} \int_{T} c_{k}^{T}(t) u_{k}(t) d t \\
& +\sum_{k=1}^{N} \int_{T} v_{k}(t) d t-\sum_{k=1}^{N} \int_{T} w_{k}(t) d t \\
= & {\left[\sum_{k=1}^{N}\left(\nu^{k}\right)^{T} \sum_{s=1}^{k} \int_{T} g_{k s}(t) u_{s}(t) d t+\sum_{k=1}^{N} \int_{T} v_{k}(t) d t-\sum_{k=1}^{N} \int_{T} w_{k}(t) d t\right] } \\
& -\left[\sum_{k=1}^{N} \sum_{s=k}^{N} \int_{T}\left(y_{s}^{0}\right)^{T} g_{s k}(t) u_{k}^{0}(t) d t+\sum_{k=1}^{N} \int_{T} v_{k}^{0}(t) d t-\sum_{k=1}^{N} \int_{T} w_{k}^{0}(t) d t\right] \\
& +\sum_{k=1}^{N} \int_{T} c_{k}(t) u_{k}^{0}(t) d t-\sum_{k=1}^{N} \int_{T} c_{k}(t) u_{k}(t) d t \\
= & {\left[\sum_{k=1}^{N}\left(\nu^{k}\right)^{T} h_{k}+\sum_{k=1}^{N} \int_{T}\left(v_{k}(t)-w_{k}(t)\right) d t\right] } \\
& -\left[\sum_{k=1}^{N}\left(y_{k}^{0}\right)^{T} h_{k}+\sum_{k=1}^{N} \int_{T}\left(v_{k}^{0}(t)-w_{k}^{0}(t)\right) d t\right] \\
& +\sum_{k=1}^{N} \int_{T} c_{k}(t) u_{k}^{0}(t) d t-\sum_{k=1}^{N} \int_{T} c_{k}(t) u_{k}(t) d t .
\end{aligned}
$$

Copyright $@$ by SIAM. Unauthorized reproduction of this article is prohibited. 
Hence the suboptimal estimate can be written in the form

$$
\beta\left(\tau_{\text {sup }}, u\right)=\beta_{\text {sup }}+\beta_{u},
$$

where

$$
\beta_{\text {sup }}=\sum_{k=1}^{N} h_{k}^{T}\left(\nu_{k}-y^{0 k}\right)+\sum_{k=1}^{N} \int_{T}\left[\left(v_{k}(t)-v_{k}^{0}(t)\right)-\left(w_{k}(t)-w_{k}^{0}(t)\right)\right] d t
$$

denotes the nonoptimality measure of the chosen support $\left\{\tau_{\text {sup }}^{k}, k \in K\right\}$ and

$$
\beta_{u}=\sum_{k=1}^{N} \int_{T} c_{k}(t)\left(u_{k}(t)-u_{k}^{0}(t)\right) d t
$$

denotes the nonoptimality measure of the given control function $\left\{u_{k}(t), t \in T, k \in\right.$ $K\}$.

Now choose the support $\tau_{\text {sup }}^{0}=\left\{\tilde{\tau}_{\text {sup }}^{k}, k \in K\right\}$ such that the corresponding collection $z_{k}^{0}=\left\{y_{k}^{0}, v_{k}^{0}, w_{k}^{0}, k \in K\right\}$ of dual variables is an optimal solution of (43)-(44). Then the support $\tau_{\text {sup }}^{0}=\left\{\tilde{\tau}_{\text {sup }}^{k}(\epsilon), k \in K\right\}$ is the one required for the given $\epsilon$-optimal control functions $\left\{u_{k}(t), k \in K\right\}$, since $\beta_{\text {sup }}=0$, and then $\beta=\beta\left(u, \tau_{\text {sup }}^{0}\right)=\beta_{u} \leq \epsilon$. Next set

$$
\begin{aligned}
& \epsilon_{k}(t)=\Delta_{k}(t)\left(u_{k}(t)+1\right), \quad t \in T_{k}^{+}, \\
& \epsilon_{k}(t)=\Delta_{k}(t)\left(u_{k}(t)-1\right), \quad t \in T_{k}^{-}, \\
& \epsilon_{k}(t)=0 \quad \text { if } \quad \Delta_{k}(t)=0, t \in T,
\end{aligned}
$$

and note from the definition of $\Delta_{k}(t)$ that

$$
\begin{aligned}
\epsilon_{k}(t)= & -\psi_{k}^{T}(t) b\left(u_{k}(t)+1\right)=\psi_{k}^{T}(t)\left(A x_{k}(t)+D x_{k-1}(t)+b(-1)\right) \\
& -\psi_{k}^{T}(t)\left(A x_{k}(t)+D x_{k-1}(t)+b u_{k}(t)\right) \quad \text { if } \psi_{k}(t) b<0, \\
\epsilon_{k}(t)= & \psi_{k}^{T}(t)\left(A x_{k}(t)+D x_{k-1}(t)+b(+1)\right) \\
& -\psi_{k}^{T}(t)\left(A x_{k}(t)+D x_{k-1}(t)+b u_{k}(t)\right) \quad \text { if } \psi_{k}(t) b>0, \\
\epsilon_{k}(t)= & 0 \quad \text { if } \quad \psi_{k}(t) b=0, \quad t \in T, \quad k \in K .
\end{aligned}
$$

Use of the Hamiltonian (35) now enables these last expressions to be written in the form

$$
\epsilon_{k}(t)=\max _{|v| \leq 1} H_{k}\left(x_{k-1}(t), x_{k}(t), \psi_{k}, v\right)-H_{k}\left(x_{k-1}(t), x_{k}(t), \psi_{k}, u_{k}(t)\right), t \in T, k \in K .
$$

Finally, noting that $\left\{u_{k}(t)\right\}$ is a suboptimal control yields

$$
\begin{aligned}
\sum_{k=1}^{N} \int_{T} \epsilon_{k}(t) d t= & \sum_{k=1}^{N} \int_{T_{k}^{+}} \Delta_{k}(t)\left(u_{k}(t)+1\right) d t \\
& +\sum_{k=1}^{N} \int_{T_{k}^{-}} \Delta_{k}(t)\left(u_{k}(t)-1\right) d t=\beta\left(u, \tau_{\text {sup }}^{0}\right)=\beta_{u} \leq \epsilon,
\end{aligned}
$$

and the proof is complete.

Copyright $@$ by SIAM. Unauthorized reproduction of this article is prohibited. 
The maximum principle now follows from this last result on setting $\epsilon=0$ as stated formally in the following corollary.

Corollary 2. The admissible control $\left\{u_{k}^{0}(t), k \in K, t \in T\right\}$ is optimal if and only if there exists a support $\left\{\tau_{\text {sup }}^{0 k}, k \in K\right\}$ such that the supporting control $\left\{u_{k}^{0}(t), \tau_{\text {sup }}^{0 k}, t \in T, k \in K\right\}$ satisfies the maximum conditions

$$
\max _{|v| \leq 1} H_{k}\left(x_{k-1}^{0}(t), x_{k}^{0}(t), \psi_{k}, v\right)=H_{k}\left(x_{k-1}^{0}(t), x_{k}^{0}(t), \psi_{k}, u_{k}^{0}(t)\right)
$$

for all $k \in K, t \in T$, where $\psi_{k}(t)$ are the corresponding solutions of (34).

4. Differentiable properties of the optimal solutions. An important aspect of the optimization theory is sensitivity analysis of optimal controls since, in practice, the system considered can be subject to disturbances or parameters in the available model can easily arise. Mathematically, perturbations can, for example, be described by some parameters in the initial data, boundary conditions, and control and state constraints. Hence it is clearly important to know how a problem solution depends on these parameters, and in this section we aim to characterize the changes in the solutions developed due to "small" perturbations in the parameters. This could, in turn, enable us to design fast and reliable real-time algorithms to correct the solutions for these effects. As shown next, the major advantage of the constructive approach developed in this paper is that the sensitivity analysis and some differential properties of the optimal controls under disturbances can be analyzed.

Suppose that disturbances influence the initial data for (1)-(4). In particular, consider the system (1)-(4) on the interval $T_{s}=[s, \alpha]$ with the initial data $x_{k}(s)=$ $z_{k}, z_{k} \in G_{k}, k \in K$, where $G_{k} \subset \mathbb{R}^{n}$ is some neighborhood of the point $x_{k}=d_{k}$ and $s$ belongs to the neighborhood $G_{0}$ of $t=0$. We also assume that the following regularity condition holds: For the given disturbance domain $G_{k}, k \in K \cup\{0\}$, the structure of the optimal control functions for the nondisturbed data is preserved; i.e., the number of switching instances together with their order is constant.

Using Theorem 1, the optimal controls $\left\{u_{k}^{0}(t, s, z), k \in K\right\}$ are determined by the supporting time instances $\tau_{k j}=\tau_{k j}(s, z), k \in K, j=1, \ldots, m$, which are dependent on the disturbances $\left(s, z_{k}\right), s \in G_{0}, z_{k} \in G_{k}, k \in K$. Here we study the differential properties of the functions $\tau_{k j}=\tau_{k j}(s, z), k \in K, j=1, \ldots, m$, and for ease of notation we set $\tau \equiv \tau(s, z)=\left\{\tau_{k j}(s, z), \quad k \in K, j=1, \ldots, m\right\}, z=\left\{z_{k}, k \in K\right\}$ in what follows.

TheOREm 3. If (1)-(4) is regular, then for any $k \in K$ and $j=1, \ldots, m$ the functions $\tau_{k j}=\tau_{k j}(s, z)$ are differentiable in the domain $G_{0} \times G_{k} \subset \mathbb{R} \times \mathbb{R}^{n}$.

Proof. Using (10)-(11) and Theorem 1 it follows immediately that the switching instances $\tau_{k j}=\tau_{k j}(s, z), k \in K, j=1, \ldots, m$, of the optimal bang-bang control $\left\{u_{k}^{0}(t, s, z), k \in K\right\}$ for (1)-(4) in this case are the solutions of the following optimization problem:

$$
\max _{\tau_{k j}} \sum_{k \in K} R_{k}(s, z) \sum_{j=1}^{m+1}(-1)^{j} \int_{\tau_{k j-1}}^{\tau_{k j}} c_{k}(t) d t
$$

subject to

$$
\sum_{l \in K} R_{l}(s, z) \sum_{j=1}^{m+1}(-1)^{j} \int_{\tau_{l j-1}}^{\tau_{l j}} g_{k l}(t) d t=h_{k}(s, z), \quad k \in K .
$$


Here the constant $R_{k}(s, z)= \pm 1$ denotes the value $(u=+1$ or $u=-1)$ of the optimal control on pass $k$ over the first control interval $t \in\left[s, \tau_{k 1}\right]$, and

$$
h_{k}(s, z)=o_{k}-\sum_{j=1}^{k} H_{k} K_{j}(\alpha) z_{k+1-j}-\int_{s}^{\alpha} H_{k} K_{k}(\alpha-t) D f(t) d t .
$$

Also it is clear that the switching instances $\tau_{k j}=\tau_{k j}(s, z)$ satisfy

$$
\tau_{k 0}<\tau_{k 1}<\tau_{k 2}<\cdots<\tau_{k m}<\tau_{k m+1}, \quad \tau_{k 0}=s, \quad \tau_{k m+1}=\alpha .
$$

Since $\left\{u_{k}^{0}, \tau_{\text {sup }}^{0}, k \in K\right\}$ is the optimal supporting control for (1)-(4) in the absence of disturbances, the optimization problem (48)-(49) has the optimal solution $\tau_{k j}^{0}, k \in$ $K, j=1, \ldots, m\}$ at $s=0, z_{k}=\alpha_{k}, k \in k, j=1, \ldots, m$. Hence there exist Lagrange multipliers $\lambda_{k}^{0} \in \mathbb{R}^{m}, k \in K$, which are not simultaneously equal to zero, such that the collection $\left\{\lambda_{k}^{0}, \tau_{k j}^{0}\right\}$ is a stationary point for the following Lagrange function associated with the optimization problem (48)-(49):

$$
\begin{aligned}
L\left(\lambda, \tau_{\text {sup }}\right)= & \sum_{k \in K} R_{k}(s, z) \sum_{j=1}^{m+1}(-1)^{j} \int_{\tau_{k j-1}}^{\tau_{k j}} c_{k}(t) d t \\
& +\sum_{k \in K} \lambda_{k}\left[\sum_{l \in K} R_{l}(s, z) \sum_{j=1}^{m+1}(-1)^{j} \int_{\tau_{l j-1}}^{\tau_{l j}} g_{k l}(t) d t-h_{k}(s, z)\right] .
\end{aligned}
$$

The well-known stationarity conditions for a Lagrange function now yield

$$
\begin{aligned}
2 R_{k}(s, z)\left[c_{k}\left(\tau_{k j}\right)+\sum_{l=k}^{N} \lambda_{l} g_{l k}\left(\tau_{k j}\right)\right] & =0, \quad j=1, \ldots, m, \quad k \in K, \\
\sum_{l=1}^{k} R_{l}(s, z) \sum_{j=1}^{m+1}(-1)^{j} \int_{\tau_{l j-1}}^{\tau_{l j}} g_{k l}(t) d t-h_{k}(s, z) & =0, \quad k \in K,
\end{aligned}
$$

with respect to the unknown $\lambda_{k}$ and $\tau_{k}(s, z), k \in K, j=1, \ldots, m$. Also the Jacobian matrix $D$ of the mapping (52) with respect to variables $\left(\lambda, \tau_{\text {sup }}\right)$ calculated at $s=0$ and $z_{k}=\alpha_{k}$ can be written in the form

$$
D=\prod_{k \in K} 2 R_{k}(0, \alpha)\left(\begin{array}{cc}
\hat{G}_{\text {sup }} & F \\
0 & \hat{G}_{\text {sup }}
\end{array}\right),
$$

where (see also (15) for the notation here) the matrix $\hat{G}_{\text {sup }}$ is given by

$$
\hat{G}_{\text {sup }}=\left(\begin{array}{cc}
g_{k j}(t), & t \in \tau_{\text {sup }}^{j} \\
j \leq k \leq N, & j=1, \ldots, N
\end{array}\right)
$$

and the matrix $F$ is formed from the derivatives of the functions $c_{k}(t), g_{k l}(t)$ evaluated at the corresponding points. By the definition of the supporting time instances we have $\operatorname{det} D \neq 0$, and by the implicit function theorem there exists a neighborhood of the point $\left(0, \alpha_{k}, k \in K\right)$ where (52) has a unique solution $\lambda=\lambda(s, z), \tau_{k j}=\tau_{k j}(s, z)$, and these functions are also differentiable. This completes the proof.

The differential properties of the optimal controls developed above can be used for sensitivity analysis and the solution of the synthesis problem considered here. In 
particular, the supporting control approach [10] can be used to produce the differential equations for the switching time functions $\tau(s, z)$ necessary to design the optimal controllers. In a similar manner to [6] it can be shown that these satisfy the following differential equations:

$$
G \frac{\partial \tau}{\partial s}+Q=\frac{\partial h}{\partial s}, \quad P \frac{\partial \tau}{\partial z}=\frac{\partial h}{\partial z}
$$

where $h(s, z)=\left(h_{1}(s, z), \ldots, h_{m}(t, s)\right)$ is an $m N \times 1$-vector given by (50) and the matrices $G, Q, P$ are defined (see [6]) by those defining the process dynamics and information associated with the disturbance-free optimal solution. For example (see also (13)), $G=\Lambda \tilde{G}_{\text {sup }}$, where the compatibly dimensioned block matrix $\Lambda$ is constructed by the disturbance-free optimal control values $u_{k}^{0}(t) ; k=1, \ldots, N$ calculated in the supporting moments $\tau_{k j}$ from $\tau_{\text {sup. }}^{0}$. Also, by Theorem 1 , these values are equivalent to the values of $\frac{d \Delta_{i}\left(\tau_{k j}\right)}{d t}$ evaluated for the corresponding indexes $i ; j ; k$, where the functions $\Delta_{i}(t) ; i=1, \ldots, N$ are designed using the switching times of the basic optimal control function. Note also that analogous differential equations can be established for the optimal values of the cost function (treated as the function $J(s, z) \equiv J(u(\tau(s, z))$ ).

Remark 5. The equations (56) are (sometimes) termed Pfaff differential equations and model an essentially distinct class of continuous $n D$ systems. The main characteristic feature of this model is that it is overdetermined (the number of equations exceeds the unknown functions). It can also be shown that if the nondegenerate assumption on the supporting control functions holds, then so do the so-called Frobenious conditions which guarantee the existence and uniqueness of solutions of the Pfaff differential equations.

5. An example. In order to demonstrate the advantages of the supporting control function approach, we give an example where, as a preliminary, it is instructive to consider the case of $N=1$ and, in particular,

$$
\max _{|u| \leq 1} J(u), J(u):=x^{(2)}(1)
$$

for

$$
\begin{aligned}
& \frac{d x^{(1)}(t)}{d t}=x^{(2)}, \quad x^{(1)}(t), x^{(2)}(t) \in \mathbb{R}, \quad t \in[s, 1], \\
& \frac{d x^{(2)}(t)}{d t}=u(t), \quad x^{(1)}(s)=z_{1}, \quad x^{(2)}(s)=z_{2},
\end{aligned}
$$

subject to the following constraints on the control signal and a terminal state constraint, respectively:

$$
|u(t)| \leq 1, \quad x^{(1)}(1)=1 / 8 .
$$

Note that here the superscript $(\cdot)$ is used to denote a particular element in the state vector.

In this case it is easy to verify that for $s=0$ and $x^{(1)}(0)=0, x^{(2)}(0)=0$ the optimal control signal is given by

$$
u^{0}(t)=-1 \quad \text { for } \quad 0 \leq t \leq 1-\sqrt{5 / 8} \quad \text { and } \quad u^{0}(t)=+1 \quad \text { for } \quad 1-\sqrt{5 / 8}<t \leq 1 .
$$


Synthesis of the optimal control can be realized using the switching instance function $\tau=\tau\left(z_{1}, z_{2}, s\right)$, which has to satisfy the following differential equations:

$$
\begin{aligned}
\frac{\partial \tau}{\partial z_{1}} & =\frac{1}{2(1-\tau)}, \\
\frac{\partial \tau}{\partial z_{2}} & =\frac{1-s}{2(1-\tau)}, \\
\frac{\partial \tau}{\partial s} & =\frac{1-s-z_{2}}{2(1-\tau)},
\end{aligned}
$$

with the initial condition

$$
\tau(0,0,0)=1-\sqrt{5 / 8}
$$

which is a particular case of (56).

The solution of this Pfaff differential system is given by

$$
\tau\left(z_{1}, z_{2}, s\right)=1-\sqrt{5 / 8+(s-1) z_{2}-z_{1}-s+s^{2} / 2} .
$$

Also, without loss of generality, assume $s=0$, and then the optimal switching function is

$$
\tau\left(z_{1}, z_{2}, 0\right)=1-\sqrt{5 / 8-z_{1}-z_{2}} .
$$

Figures 1 and 2 illustrate the form of this solution. In particular, Figure 1 shows the state-space variables together with additional variable $t$. The optimal trajectories (57)-(59) corresponding to the bang-bang control law lie on the parabolic cylinders $\left(Z_{1}\right): x^{(1)}=-\frac{1}{2}\left(x^{(2)}\right)^{2}+C_{1}+C_{2}$ and $\left(Z_{2}\right): x^{(1)}=+\frac{1}{2}\left(x^{(2)}\right)^{2}+\tilde{C}_{1}+\tilde{C}_{2}$, where the constants $C_{i}, \tilde{C}_{i}, i=1,2$, are determined by the initial data $x^{(1)}(0)=z_{1}, x^{(2)}(0)=z_{2}$. These cylinders correspond to the solutions of differential equations (58) with $u \equiv-1$ or $u \equiv+1$, respectively. It can also be shown that the admissible initial domain for which the problem can be solved is determined by the inequalities: $-\frac{3}{8} \leq z_{1}+z_{2} \leq \frac{5}{8}$. The switching manifold $Z_{h}$ is described in parametric form by

$$
\begin{aligned}
x^{(1)} & =-\frac{\left(1-\sqrt{5 / 8-z_{2}-z_{1}}\right)^{2}}{2}+z_{2}\left(1-\sqrt{5 / 8-z_{2}-z_{1}}\right)+z_{1}, \\
x^{(2)} & =-1+\sqrt{5 / 8-z_{2}-z_{1}}+z_{2}, \\
T & =1-\sqrt{5 / 8-z_{2}-z_{1}}-\frac{3}{8} \leq z_{1}+z_{2} \leq \frac{5}{8} .
\end{aligned}
$$

Finally, each optimal trajectory consists of two parts - first it evolves along the vertical parabolic cylinder $Z_{1}$ until $\tau=1-\sqrt{5 / 8-z_{2}-z_{1}}$, when it meets the switching manifold $Z_{h}$, and then immediately is switched to continue along the second vertical cylinder $Z_{2}$ to meet the target plane $x^{(1)}=1 / 8$. Figure 1 also shows the optimal trajectory in the space $R^{3}$ for zero initial data, and Figure 2 shows the projection of this trajectory onto the $x^{(1)}, x^{(2)}$ plane.

Consider now the following example where $N=2$ (again the superscript (.) is used to denote a particular element in the state or control vector on any pass):

$$
\max _{u_{1}, u_{2}} J(u), J(u):=x_{1}^{(2)}(1)+x_{2}^{(2)}(1),
$$




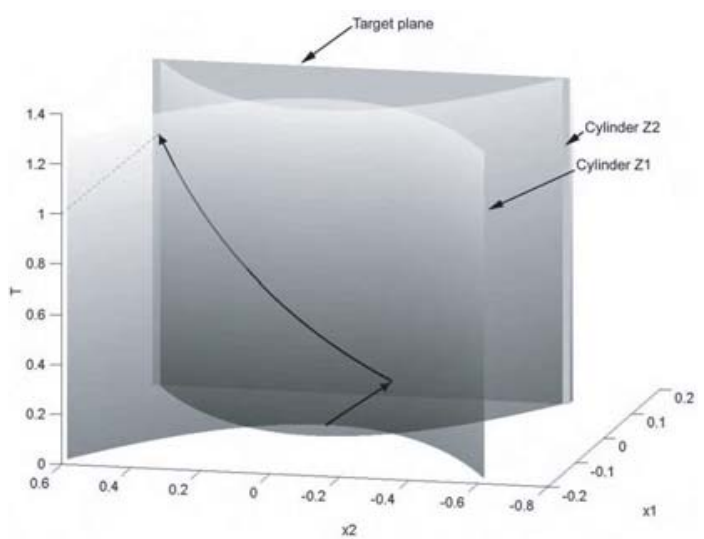

FIG. 1. Optimal control synthesis.

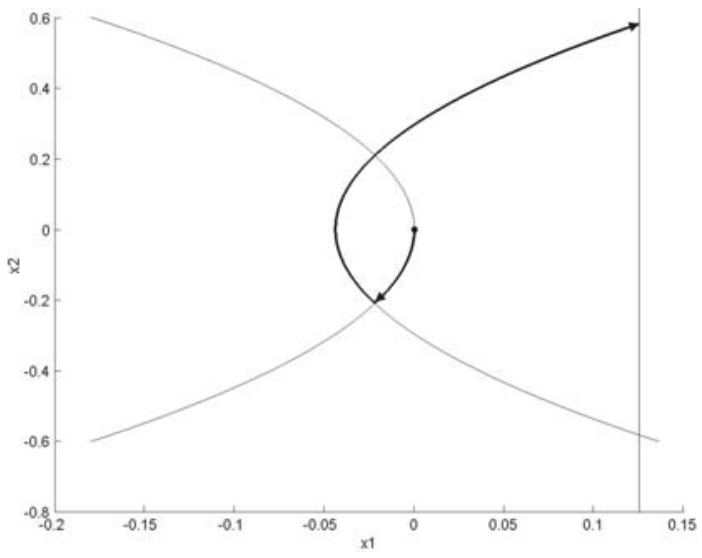

FIG. 2. Projection on the $x_{1}-x_{2}$ plane.

for the process

$$
\begin{array}{ll}
\frac{d x_{1}^{(1)}(t)}{d t}=x_{1}^{(2)}(t), & \frac{d x_{2}^{(1)}(t)}{d t}=x_{2}^{(2)}(t), t \in[s, 1], \\
\frac{d x_{1}^{(2)}(t)}{d t}=u_{1}(t), & \frac{d x_{2}^{(2)}(t)}{d t}=x_{1}^{(1)}(t)+u_{2}(t),
\end{array}
$$

with boundary conditions of the form

$$
x_{1}^{(1)}(s)=z_{1}^{(1)}, x_{1}^{(2)}(s)=z_{1}^{(2)}, x_{2}^{(1)}(s)=z_{2}^{(1)}, x_{2}^{(2)}(s)=z_{2}^{(2)},
$$

subject to

$$
x_{1}^{(1)}(1)=1 / 8, \quad x_{2}^{(1)}(1)=1 / 384,\left|u_{1}(t)\right| \leq 1,\left|u_{2}(t)\right| \leq 1 .
$$

Equivalently, we can write the problem here as

$$
\left[\begin{array}{c}
\dot{x}_{k+1}^{(1)}(t) \\
\dot{x}_{k+1}^{(2)}(t)
\end{array}\right]=\left[\begin{array}{ll}
0 & 1 \\
0 & 0
\end{array}\right]\left[\begin{array}{c}
x_{k+1}^{(1)}(t) \\
x_{k+1}^{(2)}(t)
\end{array}\right]+\left[\begin{array}{ll}
0 & 0 \\
1 & 0
\end{array}\right]\left[\begin{array}{c}
x_{k}^{(1)}(t) \\
x_{k}^{(2)}(t)
\end{array}\right]+\left[\begin{array}{l}
0 \\
1
\end{array}\right] u_{k+1}(t), k=0,1 .
$$

Without loss of generality we set $x_{0}(t)=0, t \in[s, 1]$. 
To apply the results developed here to this example we first rewrite (63)-(65) in the following integral form:

$\max _{u_{1}, u_{2}}\left\{z_{2}^{(1)}+z_{2}^{(2)}+(1-s) z_{1}^{(1)}+\frac{(1-s)^{2}}{2} z_{2}^{(2)}+\int_{s}^{1} \frac{(1-t)^{2}+2}{2} u_{1}(t) d t+\int_{s}^{1} u_{2}(t) d t\right\}$,

(67)

subject to

$$
\begin{aligned}
\int_{s}^{1}(1-t) u_{1}(t) d t= & \frac{1}{8}-z_{1}^{(1)}+(1-s) z_{1}^{(2)} \\
\int_{s}^{1}\left[\frac{(1-t)^{3}}{6} u_{1}(t)+(1-t) u_{2}(t)\right] d t= & \frac{1}{384}-z_{2}^{(1)}-(1-s) z_{2}^{(2)}-\frac{(1-s)^{2}}{2} z_{1}^{(1)} \\
& -\frac{(1-s)^{3}}{6} z_{1}^{(2)}
\end{aligned}
$$

Hence

$$
\begin{aligned}
g_{11}(t) & =1-t, \quad g_{21}(t)=\frac{(1-t)^{3}}{6}, \quad g_{22}(t)=1-t, \\
c_{1}(t) & =\frac{(1-t)^{2}+2}{2}, \quad c_{2}(t)=1,
\end{aligned}
$$

and the multipliers required to design the cocontrol function $\Delta_{i}(t), i=1,2$, can, by noting (12), be written as

$$
\nu^{(2)} g_{22}\left(\tau_{2 \text { sup }}\right)-c_{2}\left(\tau_{2 \text { sup }}\right)=0 \nu^{(1)} g_{11}\left(\tau_{1 \text { sup }}\right)+\nu^{(2)} g_{21}\left(\tau_{1 \text { sup }}\right)-c_{1}\left(\tau_{1 \text { sup }}\right)=0 .
$$

We now have that

$\Delta_{1}(t)=(1-t)\left[\frac{1}{1-\tau_{1 \text { sup }}}+\frac{1-\tau_{1 \text { sup }}}{2}-\frac{\left(1-\tau_{1 \text { sup }}\right)^{2}}{6\left(1-\tau_{2 \text { sup }}\right)}\right]+\frac{(1-t)^{3}}{6\left(1-\tau_{2 \text { sup }}\right)}-\frac{(1-t)^{2}}{2}-1$, $\Delta_{2}(t)=\frac{1-t}{1-\tau_{2 s u p}}-1$

and the problem is to find the basic optimal trajectory when all variables in (64) are zero, i.e.,

$$
s=0, x_{1}^{(1)}(0)=0, x_{1}^{(2)}(0)=0, x_{2}^{(1)}(0)=0, x_{2}^{(2)}(0)=0 .
$$

Take the supporting instances as

$$
\tau_{1 \text { sup }}=1-\sqrt{\frac{5}{8}}, \quad \tau_{2 \text { sup }}=1-\sqrt{\frac{131}{256}} .
$$

Then it follows immediately from Theorem 1 that the optimal control functions for (62)-(65) with initial data (72) are given by

$$
\begin{aligned}
& u_{1}^{0}(t)= \begin{cases}-1, & 0 \leq t<1-\sqrt{\frac{5}{8}} \\
+1, & 1-\sqrt{\frac{5}{8}} \leq t \leq 1,\end{cases} \\
& u_{2}^{0}(t)= \begin{cases}-1, & 0 \leq t<1-\sqrt{\frac{131}{256}} \\
+1, & 1-\sqrt{\frac{131}{256}} \leq t \leq 1,\end{cases}
\end{aligned}
$$

Copyright $@$ ( ) by SIAM. Unauthorized reproduction of this article is prohibited. 
and (56) gives the switching functions $\tau_{1} \equiv \tau_{1}\left(z_{1}^{(1)}, z_{1}^{(2)}, s\right), \tau_{2} \equiv \tau_{2}\left(z_{1}^{(1)}, z_{2}^{(1)}, z_{1}^{(2)}, z_{2}^{(2)}, s\right)$ as

$$
\begin{aligned}
-2 \frac{\partial \tau_{2}}{\partial s}\left(1-\tau_{2}\right)- & \frac{2\left(1-\tau_{1}\right)^{3}}{6} \frac{\partial \tau_{1}}{\partial s}=\frac{(1-s)^{2}}{2} z_{1}^{(2)}+(1-s) z_{1}^{(1)} \\
& +z_{2}^{(2)}-\frac{(1-s)^{3}}{6}-(1-s) \\
& -2 \frac{\partial \tau_{2}}{\partial z_{1}^{(1)}}\left(1-\tau_{2}\right)-\frac{\left(1-\tau_{1}\right)^{3}}{3} \frac{\partial \tau_{1}}{\partial z_{1}^{(1)}} \\
= & -\frac{(1-s)^{2}}{2}-2 \frac{\partial \tau_{2}}{\partial z_{1}^{(2)}}\left(1-\tau_{2}\right)-\frac{\left(1-\tau_{1}\right)^{3}}{3} \frac{\partial \tau_{1}}{\partial z_{1}^{(2)}} \\
= & -\frac{(1-s)^{3}}{6}-2 \frac{\partial \tau_{2}}{\partial z_{2}^{(1)}}\left(1-\tau_{2}\right)=-1, \\
-2 \frac{\partial \tau_{2}}{\partial z_{2}^{(2)}}\left(1-\tau_{2}\right)= & -(1-s),
\end{aligned}
$$

with initial conditions

$$
\tau_{1}(0,0,0)=1-\sqrt{\frac{5}{8}}, \quad \tau_{2}(0,0,0,0,0)=1-\sqrt{\frac{131}{16^{2}}} .
$$

The solutions of this differential system are

$$
\begin{aligned}
\tau_{1}\left(z_{1}^{(1)}, z_{1}^{(2)}, s\right) & =1-\sqrt{S R_{1}\left(z_{1}^{(1)}, z_{1}^{(2)}, s\right)}, \\
\tau^{(2)}\left(z_{1}^{(1)}, z_{2}^{(1)}, z_{1}^{(2)}, z_{2}^{(2)}, s\right) & =1-\sqrt{S R_{2}\left(z_{1}^{(1)}, z_{2}^{(1)}, z_{1}^{(2)}, z_{2}^{(2)}, s\right)},
\end{aligned}
$$

where

$$
S R_{1}\left(z_{1}^{(1)}, z_{1}^{(2)}, s\right)=\frac{5}{8}+(s-1) z_{1}^{(2)}-z_{1}^{(1)}-s+s^{2} / 2
$$

$S R_{2}\left(z_{1}^{(1)}, z_{2}^{(1)}, z_{1}^{(2)}, z_{2}^{(2)}, s\right)=\frac{131}{256}+\frac{2 s^{4}-8 s^{3}+59 s^{2}-102 s}{96}+\frac{-20 s^{2}+40 s-19}{48} z_{1}^{(1)}$

$$
\begin{aligned}
& -\frac{1}{12} z_{1}^{(1) 2}+\frac{4 s^{3}-12 s^{2}+11 s-3}{48} z_{1}^{(2)}+\frac{-s^{2}+2 s-1}{12} z_{1}^{(2) 2} \\
& +\frac{s z_{1}^{(1)} z_{1}^{(2)}}{6}-\frac{z_{1}^{(1)} z_{1}^{(2)}}{6}-z_{1}^{(2)}+(s-1) z_{2}^{(2)} .
\end{aligned}
$$

It easy to see that the solution of the differential equations describing the process dynamics with both $u_{1}$ and $u_{2}$ constant is

$$
\begin{aligned}
& x_{1}^{(1)}(t)=u_{1} \frac{t^{2}}{2}+t C_{1}+C_{2}, \\
& x_{1}^{(2)}(t)=u_{1} t+C_{1}, \\
& x_{2}^{(1)}(t)=u_{1} \frac{t^{4}}{24}+C_{1} \frac{t^{3}}{6}+C_{2} \frac{t^{2}}{2}+u_{2} \frac{t^{2}}{2}+t C_{3}+C_{4}, \\
& x_{2}^{(2)}(t)=u_{1} \frac{t^{3}}{6}+C_{1} \frac{t^{2}}{2}+t C_{2}+t u_{2}+C_{3}
\end{aligned}
$$

and in this case that the optimal control for pass $k=1$ coincides with that given earlier in this example.

Copyright $@$ by SIAM. Unauthorized reproduction of this article is prohibited. 
Now consider disturbances $\Omega$ such that the optimal control is preserved for the case of zero initial conditions, i.e., $u_{1}=-1$ for $t \leq \tau_{1}\left(z_{1}^{(1)}, z_{1}^{(2)}, s\right), u_{2}^{0}=-1$ for $t \leq$ $\tau_{2}\left(z_{1}^{(1)}, z_{2}^{(1)}, z_{1}^{(2)}, z_{2}^{(2)}, s\right)$, and the inequality $\tau_{1}\left(z_{1}^{(1)}, z_{1}^{(2)}, s\right)<\tau_{2}\left(z_{1}^{(1)}, z_{2}^{(1)}, z_{1}^{(2)}, z_{2}^{(2)}, s\right)$ holds. Using (77) we have that the domain $\Omega$ is described by

$$
\begin{gathered}
0 \leq \tau_{1}\left(z_{1}^{(1)}, z_{1}^{(2)}, s\right)<\tau_{2}\left(z_{1}^{(1)}, z_{2}^{(1)}, z_{1}^{(2)}, z_{2}^{(2)}, s\right) \leq 1, \\
S R_{1}\left(z_{1}^{(1)}, z_{1}^{(2)}, s\right) \geq 0, \quad S R_{2}\left(z_{1}^{(1)}, z_{2}^{(1)}, z_{1}^{(2)}, z_{2}^{(2)}, s\right) \geq 0 .
\end{gathered}
$$

To construct the solution for pass $k=2$, it is necessary to construct the switching surface $F$, which is defined by the vectors

$$
\begin{aligned}
& \left.x_{2}^{(1)}(t)\right|_{t=\tau_{2}}=x_{2}^{(1)}\left(\tau_{2}\left(z_{1}^{(1)}, z_{2}^{(1)}, z_{1}^{(2)}, z_{2}^{(2)}, s\right), \tau\right) \\
& \left.x_{2}^{(2)}(t)\right|_{t=\tau_{2}}=x_{2}^{(2)}\left(\tau_{2}\left(z_{1}^{(1)}, z_{2}^{(1)}, z_{1}^{(2)}, z_{2}^{(2)}, s\right)\right),
\end{aligned}
$$

when the parameters $z_{1}^{(1)}, z_{2}^{(1)}, z_{1}^{(2)}, z_{2}^{(2)}, s$ are members of the set $\Omega$. The parametric description of the switching surface in this case is given by

$$
\begin{aligned}
& x_{2}^{(1)}(t)=-\frac{t^{4}}{24}+C_{1} \frac{t^{3}}{6}+C_{2} \frac{t^{2}}{2}-\frac{t^{2}}{2}+t C_{3}+C_{4}, \\
& x_{2}^{(2)}(t)=-\frac{t^{3}}{6}+C_{1} \frac{t^{2}}{2}+t C_{2}-t+C_{3},
\end{aligned}
$$

where the coefficients $C_{i}$ are found from the parameters $z_{1}^{(1)}, z_{2}^{(1)}, z_{1}^{(2)}, z_{2}^{(2)}, s$.

6. Conclusions. In this paper the supporting control functions approach has been applied to study an optimal control problem for differential linear repetitive processes. The main contribution is the development of constructive necessary and sufficient optimality conditions in forms which can be effectively used for the design of numerical algorithms. The iterative method developed in this work is based on the principle of decrease of the suboptimality estimate; i.e., the iteration $\left\{\tau_{\text {sup }}^{k}, u_{k}(t), k=\right.$ $1, \ldots, N\} \rightarrow\left\{\hat{\tau}_{\text {sup }}^{k}, \hat{u}_{k}(t), k=1, \ldots, N\right\}$ is performed in such a way as to achieve $\beta\left(\hat{\tau}_{\text {sup }}, \hat{u}\right)<\beta\left(\tau_{\text {sup }}, u\right)$. Also this procedure can be separated into two stages: (i) transformation of the admissible control functions $\left\{u_{k}(t), k=1, \ldots, N\right\} \rightarrow\left\{\hat{u}_{k}(t), k=\right.$ $1, \ldots, N\}$, which decreases the nonoptimality measure of the admissible controls $\beta(\hat{u})<\beta(u)$, and (ii) variation of the support $\left\{\tau_{\text {sup }}^{k}, k=1, \ldots, N\right\} \rightarrow\left\{\hat{\tau}_{\text {sup }}^{k}, k=\right.$ $1, \ldots, N\}$ to again decrease the nonoptimality measure of the support, i.e., $\beta\left(\hat{\tau}_{\text {sup }}\right)<$ $\beta\left(\tau_{\text {sup }}\right)$. These transformations involve, in effect, the duality theory for the problems defined in this work by (1)-(4) and (43)-(44) and exploit the $\epsilon$-optimality conditions also developed in this work. These results are the first in this general area, and work is currently proceeding in a number of followup areas. One such area is sensitivity analysis of optimal control in the presence of disturbances, where in the case of the ordinary linear control systems some work on this topic can be found in, for example, [12].

\section{REFERENCES}

[1] N. Amann, D. H. Owens, And E. Rogers, Iterative learning control using optimal feedforward and feedback actions, Internat. J. Control, 69 (1996), pp. 277-293.

[2] T. Al-Towaim, A. D. Barton, P. L. Lewin, E. Rogers, and D. H. Owens, Iterative learning control - 2D systems from theory to application, Internat. J. Control, 77 (2004), pp. 877893. 
[3] S. Arimoto, S. Kawamura, and F. Miyazaki, Bettering operations of robots by learning, J. Robotic Systems, 1 (1984), pp. 123-140.

[4] M. Bisiacco And E. Fornasini, Optimal control of two-dimensional systems, SIAM J. Control Optim., 28 (1990), pp. 582-601.

[5] W. D. Collins, Controllability and canonical forms for multipass systems described by the ordinary differential equations, IMA J. Math. Control Inform., 1 (1984), pp. 1-25.

[6] M. P. Dymkov and S. Gnevko, Continuous linear programming with desturbed parameters, Reports of the Academy Sciences of Belarus, Series Physics and Mathematics, 4 (1983), pp. 8-14 (in Russian).

[7] C. Du And L. XIE, $H_{\infty}$ Control and Filtering of Two-Dimensional Systems, Lecture Notes in Control and Inform. Sci. 278, Springer-Verlag, Berlin, 2002.

[8] E. Fornasini And G. Marchesini, Doubly indexed dynamical systems: State-space models and structual properties, Math. Systems Theory, 12 (1978), pp. 59-72.

[9] K. Fujimoto, T. Horiuchi, And T. Sugie, Optimal control of Hamiltonian systems with input constraints via iterative learning, in Proceedings of the 42nd IEEE Conference on Decision and Control, 2003, pp. 4387-4392.

[10] R. Gabasov and F. M. Kirillova, Software Optimization, Plenum Press, New York, 1988.

[11] R. Gabasov, F. M. Kirillova, and S. V. Prischepova, Optimal Feedback Control, Lecture Notes in Control and Inform. Sci. 207, Springer-Verlag, Berlin, 1995.

[12] O. I. Kostyukova, Parametric optimal control problem with variable index, Comput. Math. Math. Phys., 43 (2003), pp. 26-41; (Zh. Vychis. Mat. i Mat. Fiz., 2003, 43(1), pp. 26-41, in Russian).

[13] K. L. Moore, Y. Chen, And V. BAhL, Monotonically convergent iterative learning control for linear discrete-time systems, Automatica, 41 (2005), pp. 1529-1537

[14] D. H. Owens, N. Amann, E. Rogers, And M. French, Analysis of linear iterative learning control schemes - A 2D systems/repetitive processes approach, Multidimensi. Syst. Signal Process., 11 (2000), pp. 125-177.

[15] P. D. RoberTs, Numerical investigations of a stability theorem arising from 2-dimensional analysis of an iterative optimal control algorithm, Multidimens. Syst. Signal Process., 11 (2000), pp. 109-124.

[16] R. P. RoEsser, A discrete state space model for linear image processing, IEEE Trans. Automat. Control, 20 (1975), pp. 1-10.

[17] E. Rogers And D. H. Owens, Stability Analysis for Linear Repetitive Processes, Lecture Notes in Control and Inform. Sci. 175, Springer-Verlag, Berlin, 1992.

[18] M. SebeK and F. J. Kraus, Stochastic LQ-optimal control for 2-D systems, Multidimens. Syst. Signal Process., 6 (1995), pp. 275-285.

[19] H. D. Tuan, P. Apkarian, T. Q. Nguyen, and T. Narikiyo, Robust Mixed $H_{2} / H_{\infty}$ filtering of 2-D systems, IEEE Trans. Signal Process., 50 (2002), pp. 1759-1771.

[20] J.-X. XU, Y. TAN, AND T.-H. LEE, Iterative learning control design based on composite energy function with input saturation, Automatica, 40 (2004), pp. 1371-1377.

Copyright $@$ by SIAM. Unauthorized reproduction of this article is prohibited. 